\title{
Impact of recent COMPASS data on polarized parton distributions and structure functions
}

\author{
M. Salimi-Amiri, ${ }^{*}$ A. Khorramian, ${ }^{\dagger}$ and H. Abdolmaleki ${ }^{\ddagger}$ \\ Faculty of Physics, Semnan University, 35131-19111 Semnan, Iran \\ F. I. Olness ${ }^{\S}$ \\ Department of Physics, Southern Methodist University, Dallas, Texas 75275-0175, USA
}

(Received 8 May 2018; published 24 September 2018)

\begin{abstract}
We perform a new extraction of polarized parton distribution functions (PPDFs) from the spin structure function experimental data in the fixed-flavor number scheme. In this analysis, we include recent proton and deuteron spin structure functions obtained by the COMPASS Collaboration. We examine the impact of the new COMPASS proton and deuteron data on the polarized parton densities and compare with results from our previous study (KATAO PPDFs), which used the Jacobi polynomial approach. We find the extracted PPDFs of the proton, neutron, and deuteron structure functions are in very good agreement with the experimental data. The results for extracted PPDFs are also compared with available theoretical models from the literature.
\end{abstract}

DOI: 10.1103/PhysRevD.98.056020

\section{INTRODUCTION}

One of the principal goals of quantum chromodynamics (QCD) has been the detailed investigation of the spin structure of the nucleon and nuclei, as well as the determination of the partonic composition of their spin projections. The extraction of polarized or spin-dependent parton distribution functions has been recognized as a longstanding issue of physical interest [1,2], and theoretical studies on the spin structure of the nucleon have been discussed extensively in several reviews [3-7].

Determinations of polarized parton distribution functions (PPDFs) with an estimate of their uncertainties have been presented in multiple studies [8-43]. The variation among these PPDF sets can be due to a number of factors, including the choice of experimental data sets and the form of the parametrization and uncertainty calculation, as well as the details of the QCD analysis such as the treatment of heavy quarks or higher-twist corrections.

The results from various calculations can lead to a wide range of expectations for the polarized observables; hence, it

\footnotetext{
*Maryam.salimi.amiri@semnan.ac.ir

†horramiana@semnan.ac.ir

*Abdolmaleki@semnan.ac.ir

§olness@smu.edu
}

Published by the American Physical Society under the terms of the Creative Commons Attribution 4.0 International license. Further distribution of this work must maintain attribution to the author(s) and the published article's title, journal citation, and DOI. Funded by SCOAP ${ }^{3}$. is illuminating to compare the results of different methodologies to the experimental measurements. In our previous analysis, we performed the detailed $\mathrm{pQCD}$ analysis of PPDFs using the orthogonal Bernstein and Jacobi polynomial methods at next-to-leading order (NLO) [44-46]. Other theoretical studies implementing a QCD analysis on the spin structure of the nucleon using orthogonal polynomials have been reported in Refs. [47-50]. Thus, one goal of our investigation is to revisit this topic using a Mellin moment approach instead of the orthogonal polynomial approach.

For the present study, we will focus on the polarized structure functions of the nucleon $g_{1}^{p, n, d}\left(x, Q^{2}\right)$, which play an important role in the behavior of PPDFs. Polarized deep inelastic scattering (DIS) lepton-nucleon scattering has been measured by DESY [51-53], SLAC [54-60], COMPASS [61-65], CLAS [66], and JLAB [67].

Recently, the COMPASS Collaboration [68,69] extracted new DIS measurements of the polarized proton and deuteron structure functions for the regions $0.0035<$ $x<0.575,1.03<Q^{2}<96.1 \mathrm{GeV}^{2}$ and $0.0045<x<0.569$, $1.03<Q^{2}<74.1 \mathrm{GeV}^{2}$. Thus, we will combine the data sets used in Ref. [44] with the COMPASS16 and COMPASS17 data sets to extract improved polarized structure functions and PPDFs.

The plan of this paper is as follows: In Sec. II, we review the theoretical framework and basic formalism of the polarized structure function analysis based on the inverse Mellin technique. In Sec. III, we outline the parametrization of PPDFs and the selection of the data sets. In Sec. IV, we present the structure functions, PPDFs, and moments 
obtained in our fit, and compare these both to our earlier KATAO fit (using orthogonal polynomials) as well as other results from the literature; this also includes an evaluation of the impact of the new COMPASS data sets. Finally, in Sec. V, we provide a summary and concluding remarks.

\section{THEORETICAL FORMALISM}

The QCD formalism allows us to express the spindependent nucleon structure function $g_{1}\left(x, Q^{2}\right)$ in terms of a Mellin convolution of the polarized nonsinglet $\delta q_{i}^{\mathrm{NS}}$, the polarized singlet $\delta \Sigma$, and the polarized gluon $\delta g$ distributions with the corresponding Wilson coefficient functions $\delta C_{q}^{\mathrm{NS}}, \delta C_{q}^{S}$, and $\delta C_{g}$. The polarized structure function is then given by [4]

$$
\begin{aligned}
g_{1}\left(x, Q^{2}\right)= & \frac{1}{2} \sum_{j=1}^{n_{f}} e_{j}^{2}\left\{\delta q_{j}^{\mathrm{NS}} \otimes\left[1+\frac{\alpha_{s}}{2 \pi} \delta C_{q}^{\mathrm{NS}}\right] \frac{1}{n_{f}} \delta \Sigma\right. \\
& \left.\otimes\left[1+\frac{\alpha_{s}}{2 \pi} \delta C_{q}^{S}\right]+\frac{\alpha_{s}}{2 \pi} \delta g\left(x, Q^{2}\right) \otimes \delta C_{g}\right\},
\end{aligned}
$$

where $e_{j}$ denotes the charge of the $j$ th quark flavor, $n_{f}$ is the number of light flavors, $x$ is the Bjorken variable, $Q^{2}=-q^{2}$ is the four-momentum transfer, and the symbol $\otimes$ denotes the Mellin convolution. The coefficient functions $\delta C_{i}$ which we use in the present analysis are calculated in the $\overline{\mathrm{MS}}$ scheme at next-to-leading order [70-73]; in particular, we make use of the Pegasus routines [74]. The spin-dependent flavor nonsinglet distribution $\delta q_{j}^{\mathrm{NS}}$ evolves independently, while the spin-dependent singlet $\delta \Sigma$ and gluon distributions $\delta g$ are coupled in the QCD evolution.

In the above equation, the polarized nonsinglet and singlet PPDFs are expressed by the individual spindependent quark flavor contributions as

$$
\begin{gathered}
\delta \Sigma=\sum_{j=1}^{n_{f}}\left[\delta q_{j}+\delta \bar{q}_{j}\right], \\
\delta q_{j}^{\mathrm{NS}}=\delta q_{j}+\delta \bar{q}_{j}-\frac{1}{n_{f}} \delta \Sigma,
\end{gathered}
$$

where $\delta q_{j}$ is the polarized quark distribution function of the $j$ th light flavor.

In our fits, we will take the strong coupling constant $\alpha_{s}\left(Q_{0}^{2}\right)$ at initial scale $Q_{0}^{2}$ as a free parameter to be fit. The evolution of the strong coupling constant $\alpha_{s}\left(Q^{2}\right)$ can be obtained from the QCD renormalization group equation and is determined by the $\beta$ function, $\beta\left(Q^{2}\right)$ :

$$
\frac{d \alpha_{s}\left(Q^{2}\right)}{d \log \left(Q^{2}\right)}=\beta\left(Q^{2}\right)=-\beta_{0} \alpha_{s}^{2}\left(Q^{2}\right)-\beta_{1} \alpha_{s}^{3}\left(Q^{2}\right)+O\left(\alpha_{s}^{4}\right) .
$$

Here we have expanded the $\beta$ function in powers of $\alpha_{s}$ out to NLO, and the first two coefficients can be computed in the $\overline{\mathrm{MS}}$ scheme to be $\beta_{0}=11-\frac{2}{3} n_{f}$ and $\beta_{1}=102-\frac{38}{3} n_{f}$. Thus, given the value of $\alpha_{s}\left(Q_{0}^{2}\right)$ at the initial scale $Q_{0}^{2}$, we can numerically solve the differential equation in Eq. (4) for any $Q^{2}$ scale [74]. For the present analysis, we will work in the FFNS with $n_{f}=3$ light partonic flavors $\{u, d, s\}$.

For our fit, we will use the spin-dependent proton, neutron, and deuteron structure functions. The spindependent deuteron structure function $x g_{1}^{d}\left(x, Q^{2}\right)$ can be represented in terms of the proton and neutron structure functions, $x g_{1}^{p}\left(x, Q^{2}\right)$ and $x g_{1}^{n}\left(x, Q^{2}\right)$, using the relation

$$
x g_{1}^{d}\left(x, Q^{2}\right)=\frac{1}{2}\left(1-\frac{3}{2} \omega_{D}\right)\left[x g_{1}^{p}\left(x, Q^{2}\right)+x g_{1}^{n}\left(x, Q^{2}\right)\right],
$$

where $\omega_{D}=0.05 \pm 0.01$ is the $D$-state wave probability for the deuteron [75].

For comparison with the data, we will need to compute the PPDFs and structure functions at a variety of $Q^{2}$ scales. The evolution in $Q^{2}$ is performed using the well-known DGLAP collection of integrodifferential evolution equations [76,77], which can be solved analytically after a conversion from $x$ space to Mellin $N$-moment space.

The $N$ th Mellin moments of the spin-dependent parton densities $\delta f(x)$ are defined to be

$$
\delta f(N)=\int_{0}^{1} x^{N-1} \delta f(x) d x .
$$

The Mellin transform will decompose the convolution of parton densities $\delta f(x)$ of Eq. (1) into a product of Mellin moments:

$$
[f \otimes g](N) \equiv \int_{0}^{1} d x^{n-1} \int_{x}^{1} \frac{d y}{y} f\left(\frac{x}{y}\right) g(y)=f(N) g(N) .
$$

To invert the Mellin transform, the argument $N$ is analytically continued to the complex plane. Note that the basic method of solving the spin-dependent nonsinglet, singlet, and gluon evolution equations in Mellin space is reported in the literature in detail $[71,72,78,79]$.

The solution of the flavor nonsinglet, singlet, and gluon evolution equations at NLO are given by

$$
\begin{aligned}
& \delta q_{j}^{\mathrm{NS}}\left(N, Q^{2}\right) \\
& =\left(\frac{a_{s}}{a_{0}}\right)^{-P_{\mathrm{NS}}^{(0)} / \beta_{0}}\left[1-\frac{1}{\beta_{0}}\left(a_{s}-a_{0}\right)\left(\delta P_{\mathrm{NS}}^{(1)}-\frac{\beta_{1}}{\beta_{0}} P_{\mathrm{NS}}^{(0)}\right)\right] \\
& \quad \times \delta q_{j}^{\mathrm{NS}}\left(N, Q_{0}^{2}\right),
\end{aligned}
$$




$$
\begin{aligned}
\left(\begin{array}{c}
\delta \Sigma\left(N, Q^{2}\right) \\
\delta g\left(N, Q^{2}\right)
\end{array}\right)= & {\left[1+a_{s} U_{1}(N)\right] L\left(N, a_{s}, a_{0}\right)\left[1-a_{0} U_{1}(N)\right] } \\
& \times\left(\begin{array}{c}
\delta \Sigma\left(N, Q_{0}^{2}\right) \\
\delta g\left(N, Q_{0}^{2}\right)
\end{array}\right)
\end{aligned}
$$

where $a_{s} \equiv a_{s}\left(Q^{2}\right), a_{0}=a_{s}\left(Q_{0}^{2}\right) / 4 \pi$, and $\delta P_{\mathrm{NS}}^{(0)}$ and $\delta P_{\mathrm{NS}}^{(1)}$ denote the LO and NLO nonsinglet splitting functions. Here, the matrices $U_{1}$ and $L$ are evolution matrices; for some details, see Ref. [79].

Given the initial PPDFs at input scale $Q_{0}^{2}$, Eqs. (6) and (7) yield the distributions $\delta q^{\mathrm{NS}}\left(N, Q^{2}\right), \delta \Sigma\left(N, Q^{2}\right)$, and $\delta g\left(N, Q^{2}\right)$ in Mellin $N$ space for any scale. We can then transform back to $x$ space to obtain $\delta f(x)$ by performing a contour integral in the complex plane [80]:

$$
\delta f(x)=\frac{1}{\pi} \int_{0}^{\infty} d z \operatorname{Im}\left[\exp (i \phi) x^{-c(z)} \delta f[c(z)]\right],
$$

where we choose $c(z)=1.1+\rho[\cos (3 \pi / 4)+i \sin (3 \pi / 4)]$. The basic framework of this method is described in the literature $[30,37,80]$. The resulting $\delta f(x)$ for all PPDFs depends on the initial value of $\alpha_{s}\left(Q_{0}^{2}\right)$ and unknown parameters of the spin-dependent parton distributions; we will now discuss our parametrization form.

\section{INPUT PARAMETRIZATION AND DATA SETS}

To study the impact of the recent COMPASS16 [68] and COMPASS17 [69] data on the spin-dependent parton distribution functions, we will start by comparing to our previous KATAO [44] results; hence, our initial parametrization and $\chi^{2}$ minimization will be based on this work.

\section{A. Parametrization of the polarized parton densities}

For the parametrization of the spin-dependent parton densities in $x$ space at our initial scale $Q_{0}^{2}=4 \mathrm{GeV}^{2}$,

$$
x \delta q_{j}\left(x, Q_{0}^{2}\right)=\eta_{j} \mathcal{A}_{j} x^{\alpha_{j}}(1-x)^{\beta_{j}}\left(1+\gamma_{j} x\right) .
$$

The free parameters are $\left\{\eta_{j}, \alpha_{j}, \beta_{j}, \gamma_{j}\right\}$, and we use the common notation $\delta q_{j}=\left\{\delta u_{v}, \delta d_{v}, \delta \bar{q}, \delta g\right\}$ for the partonic flavors up-valence, down-valence, sea, and gluon. In this functional form, the terms $x^{\alpha_{j}}$ and $(1-x)^{\beta_{j}}$ control the low$x$ and large- $x$ behavior of the parton densities, respectively. The $\left(1+\gamma_{j} x\right)$ factor controls the intermediate $x$. The maximal number of parameters which should be fitted for each flavor component is four $\left\{\eta_{j}, \alpha_{j}, \beta_{j}, \gamma_{j}\right\}$, and there are four flavor components $\left\{\delta u_{v}, \delta d_{v}, \delta \bar{q}, \delta g\right\}$; this yields a total of 16 degrees of freedom, but we will introduce some constraints to reduce the number of free parameters in order to achieve a stable and reliable minimum.

The $\mathcal{A}_{j}$ and $\eta_{j}$ parameters are not independent. Since the first moment of polarized parton densities plays an important

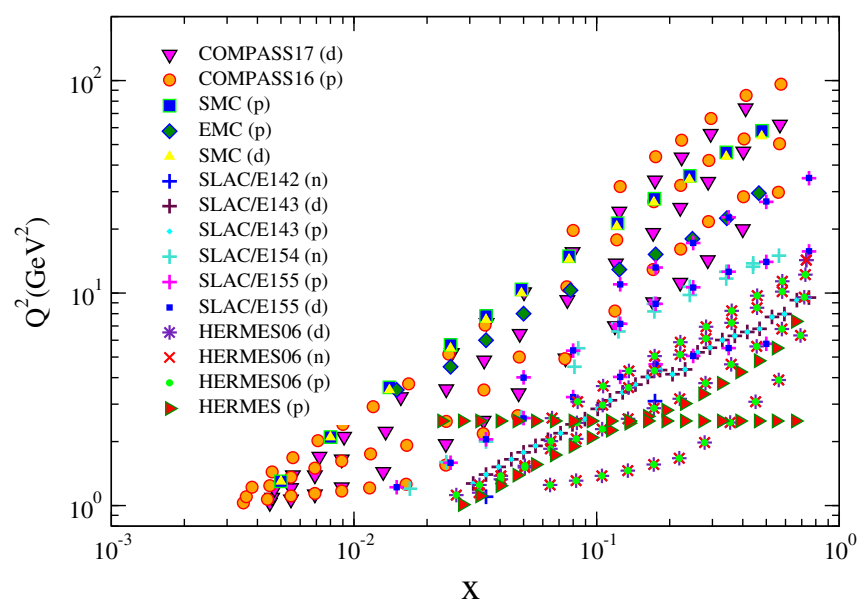

FIG. 1. Experimental data sets used in our fit of proton, deuteron, and neutron structure functions in the $\left\{x, Q^{2}\right\}$ plane.

role, the normalization constants $\mathcal{A}_{j}$ are selected such that $\eta_{j}$ are the first moments of spin dependent of parton densities $\delta q_{j}\left(x, Q_{0}^{2}\right)$; specifically, $\eta_{j}=\int_{0}^{1} d x \delta q_{j}\left(x, Q_{0}^{2}\right)$. Thus, the normalization factors $\mathcal{A}_{j}$ can be computed to be

$$
\frac{1}{\mathcal{A}_{j}}=\left(1+\gamma_{j} \frac{\alpha_{j}}{\alpha_{j}+\beta_{j}+1}\right) \mathcal{B}\left(\alpha_{j}, \beta_{j}+1\right)
$$

where $\mathcal{B}(m, n)$ is the Euler $\beta$ function.

We will presume a $S U(3)$ flavor symmetry such that $\delta \bar{q} \equiv \delta \bar{u}=\delta \bar{d}=\delta s=\delta \bar{s}$. As we mentioned before, by including only inclusive DIS data in the QCD fit, it is not possible to separate polarized quarks from polarized antiquarks. In fact, inclusive polarized DIS data constrain the total polarized quark and antiquark combinations. ${ }^{1}$ Thus, we will focus on the PPDF combinations $(\delta q+\delta \bar{q})$ as displayed in Fig. 5.

Using the above results, we can analytically compute the Mellin $N$-space transform of the polarized parton densities at the input scale of $Q_{0}^{2}$ :

$$
\begin{aligned}
\delta q_{j}\left(N, Q_{0}^{2}\right)= & \int_{0}^{1} x^{N-1} \delta q_{j}\left(x, Q_{0}^{2}\right) d x \\
= & \eta_{j} \mathcal{A}_{j}\left(1+\gamma_{j} \frac{N-1+\alpha_{j}}{N+\alpha_{j}+\beta_{j}}\right) \\
& \times B\left(N-1+\alpha_{j}, \beta_{j}+1\right) .
\end{aligned}
$$

The first moments of the polarized valence distribution, $\delta u_{v}$ and $\delta d_{v}$, can be fixed by utilizing the parameters $F$ and $D$ as measured in neutron and hyperon $\beta$ decays [81,82]. In fact,

\footnotetext{
${ }^{1}$ In Ref. [80], we reported the results of QCD analysis using polarized DIS and semi-inclusive DIS (SIDIS) asymmetry world data, and we extracted the PPDFs considering a light sea-quark decomposition.
} 
TABLE I. Data sets for polarized DIS structure functions used in our QCD analysis inclusively covering $0.0035 \leq$ $x \leq 0.75$ and $1 \leq \mathrm{Q}^{2} \leq 96.1 \mathrm{GeV}^{2}$. For each experiment we provide the $x$ and $\mathrm{Q}^{2}$ ranges, the number of data points, and the fitted normalization shifts $\mathcal{K}_{i}$.

\begin{tabular}{|c|c|c|c|c|c|c|}
\hline \multirow[b]{2}{*}{ Experiment } & \multirow[b]{2}{*}{ Reference } & \multicolumn{2}{|c|}{ Data } & \multirow[b]{2}{*}{$x$ range } & \multirow{2}{*}{$\frac{\mathrm{Q}^{2} \text { range }}{\left(\mathrm{GeV}^{2}\right)}$} & \multirow[b]{2}{*}{$\mathcal{K}_{i}$} \\
\hline & & Type & \# data points & & & \\
\hline HERMES & {$[52,53]$} & DIS $\left(g_{1}^{p}\right)$ & 39 & $0.028-0.66$ & $1.01-7.36$ & $\overline{1.000}$ \\
\hline HERMES06 & [51] & DIS $\left(g_{1}^{p}\right)$ & 51 & $0.026-0.731$ & $1.12-14.29$ & 0.999 \\
\hline SLAC/E143 & [57] & DIS $\left(g_{1}^{p}\right)$ & 28 & $0.031-0.749$ & $1.27-9.52$ & 0.999 \\
\hline SLAC/E155 & {$[60]$} & DIS $\left(g_{1}^{p}\right)$ & 24 & $0.015-0.750$ & $1.22-34.72$ & 1.023 \\
\hline SMC & [62] & DIS $\left(g_{1}^{p}\right)$ & 12 & $0.005-0.480$ & $1.30-58.0$ & 1.000 \\
\hline EMC & [61] & DIS $\left(g_{1}^{p}\right)$ & 10 & $0.015-0.466$ & $3.50-29.5$ & 1.011 \\
\hline COMPASS10 & [63] & DIS $\left(g_{1}^{p}\right)$ & 15 & $0.005-0.568$ & $1.10-62.10$ & 0.993 \\
\hline COMPASS16 & [68] & DIS $\left(g_{1}^{p}\right)$ & 51 & $0.0035-0.575$ & $1.03-96.1$ & 1.000 \\
\hline Proton & & & 230 & & & \\
\hline HERMESO6 & [51] & DIS $\left(g_{1}^{d}\right)$ & 51 & $0.026-0.731$ & $1.12-14.29$ & 0.997 \\
\hline SLAC/E143 & [57] & DIS $\left(g_{1}^{d}\right)$ & 28 & $0.031-0.749$ & $1.27-9.52$ & 0.998 \\
\hline SLAC/E155 & {$[58,59]$} & DIS $\left(g_{1}^{d}\right)$ & 24 & $0.015-0.750$ & $1.22-34.79$ & 0.999 \\
\hline SMC & [62] & DIS $\left(g_{1}^{d}\right)$ & 12 & $0.005-0.479$ & $1.30-54.80$ & 0.999 \\
\hline COMPASS17 & [69] & DIS $\left(g_{1}^{d}\right)$ & 43 & $0.0045-0.569$ & $1.03-74.1$ & 1.001 \\
\hline Deuteron & & & 158 & & & \\
\hline HERMES & {$[52,53]$} & DIS $\left(g_{1}^{n}\right)$ & 9 & $0.033-0.464$ & $1.22-5.25$ & 0.999 \\
\hline HERMES06 & [51] & DIS $\left(g_{1}^{n}\right)$ & 51 & $0.026-0.731$ & $1.12-14.29$ & 1.000 \\
\hline SLAC/E142 & [54] & DIS $\left(g_{1}^{n}\right)$ & 8 & $0.035-0.466$ & $1.10-5.50$ & 0.999 \\
\hline SLAC/E154 & [56] & DIS $\left(g_{1}^{n}\right)$ & 17 & $0.017-0.564$ & $1.20-15.00$ & 0.999 \\
\hline Neutron & & & 85 & & & \\
\hline Total & & & 473 & & & \\
\hline
\end{tabular}

$q_{3}$ and $q_{8}$ are the nonsinglet combinations of the polarized parton densities:

$$
\begin{gathered}
\delta q_{3}=(\delta u+\delta \bar{u})-(\delta d+\delta \bar{d}) \\
\delta q_{8}=(\delta u+\delta \bar{u})+(\delta d+\delta \bar{d})-2(\delta s+\delta \bar{s}) .
\end{gathered}
$$

The first moments of the above distributions are found to be

$$
\begin{aligned}
& \int_{0}^{1} d x \delta q_{3}=\eta_{u_{v}}-\eta_{d_{v}}=F+D \\
& \int_{0}^{1} d x \delta q_{8}=\eta_{u_{v}}+\eta_{d_{v}}=3 F-D
\end{aligned}
$$

Using $F=0.464 \pm 0.008$ and $D=0.806 \pm 0.008$ from the literature [31,83], we find the first moments of $\delta u_{v}$ and $\delta d_{v}$ to be $\eta_{u_{v}}=+0.928 \pm 0.014$ and $\eta_{d_{v}}=-0.342 \pm 0.018$ in our QCD fit, we will fix $\left\{\eta_{u_{v}}, \eta_{d_{v}}\right\}$ to these central values. The first moments of $\delta \bar{q}$ and $\delta g$ do not have prior constraints, and these will be determined in the fit by the free parameters $\eta_{\bar{q}}$ and $\eta_{g}$.

The above value for the octet axial charge assumes a good $S U(3)$ symmetry. It was noted in Refs. [84,85] that this symmetry can be broken by about $20 \%$, which would then yield $F \sim 0.43$ and $D \sim 0.84$, and thus $\eta_{u_{v}} \sim+0.865$ and $\eta_{d_{v}} \sim-0.405$. We have also run our fit with these modified values and observed that the variation due to these changes is small and well within our PPDF uncertainties.

The factor of $\left(1+\gamma_{j} x\right)$ in Eq. (9) provides flexibility to the parametrization in the intermediate $x$ region. This flexibility is beneficial for fitting the polarized valence distributions $\delta u_{v}, \delta d_{v}$. In contrast, we find that the parameters $\gamma_{\bar{q}}$ and $\gamma_{g}$ have a very mild impact on the fit, and it is sufficient to set them to zero and remove these degrees of freedom. (We note that the QCD analysis of polarized SIDIS data [80] is sensitive to the $\gamma_{\bar{q}}$ and $\gamma_{g}$ parameters.)

We have now reduced the number of free parameters from 16 to 12 . Preliminary fits indicate that some of the parameters such as $\left\{\gamma_{u_{v}}, \gamma_{d_{v}}, \beta_{\bar{q}}, \gamma_{g}\right\}$ are very weakly constrained by the present data set and have very large uncertainties. In fact, the precision of the data which we used is not high enough to constrain these mentioned parameters sufficiently. We found that altering them within these uncertainties does not obtain a significant change of $\chi^{2}$. Therefore, we will also fix the values of these parameters, and we now have eight free parameters remaining for the PPDFs, in addition to the QCD coupling constant $\alpha_{s}\left(Q_{0}^{2}\right)$ to fit from the data. 


\section{B. Overview of experimental data set}

The notable advances of the experimental data of inclusive polarized deep inelastic scattering on nucleons in recent years allows us to perform an improved QCD analysis of polarized structure functions in order to discern the spin-dependent partonic structure of the nucleon. For our analysis, we will include spin structure function data on protons from HERMES [51-53], E143 [57], E155 [60], SMC [62], EMC [61], and COMPASS [63,68]; on deuterons from HERMES [51], E143 [57], E155 [58,59], SMC [62], and COMPASS [69]; and on neutrons from HERMES [51-53], E142 [54], and E154 [56]. This data set includes the recent proton data from COMPASS16 [68] (51 points), and the recent deuteron data from COMPASS17 [69] (43 points). This gives us a total of 473 experimental data points spanning a kinematic range of $0.0035<x<0.75$ and $1<Q^{2}<$ $96.1 \mathrm{GeV}^{2}$; these are displayed in Fig. 1, and the detailed information and references are summarized in Table I.

In this analysis, we will evolve the PPDFs from the initial scale $Q_{0}^{2}=4 \mathrm{GeV}^{2}$ up to arbitrary scales to compare our theoretical predictions with the data across the full kinematic range. We construct a global $\chi^{2}$ function using the experimental measurements $g_{1}^{\text {Exp }}$, the experimental uncertainty (statistical and systematic added in quadrature)
$\Delta g_{1}^{\text {Exp }}$, and theoretical prediction $g_{1}^{\text {Theory }}$. Our $\chi^{2}$ is constructed as follows:

$$
\begin{aligned}
\chi_{\text {global }}^{2} & =\sum_{i=1}^{n^{\text {Exp }}} w_{i} \chi_{i}^{2} \\
& =\sum_{i=1}^{n^{\text {Exp }}} w_{i}\left[\frac{\left(\mathcal{K}_{i}-1\right)^{2}}{\left(\Delta \mathcal{K}_{i}\right)^{2}}+\sum_{j=1}^{n^{\text {Data }}}\left(\frac{\mathcal{K}_{i} g_{1, j}^{\text {Exp }}-g_{1, j}^{\text {Theory }}}{\mathcal{K}_{i} \Delta g_{1, j}^{\text {Exp }}}\right)^{2}\right],
\end{aligned}
$$

where the $i$ index sums over all experimental data sets, and in each experimental data set the $j$ index sums over all data points. We introduce a weight $w_{i}$ which allows us to apply separate weights to different experimental data sets; for the present analysis we choose all weights to be unity, $w_{i}=1$.

These data sets include statistical and systematic errors which we combine in quadrature. There is also a normalization for each experiment $\mathcal{K}_{i}$ and an associated uncertainty $\Delta \mathcal{K}_{i}$. The normalization shifts $\mathcal{K}_{i}$ are fitted at the start of our procedure, and then fixed. We present these values in Table I and find that all the $\mathcal{K}_{i}$ shifts are less than $1 \%$ except for a single value; for the SLAC/E155 experiment, we find $\mathcal{K}_{i}=1.024$.
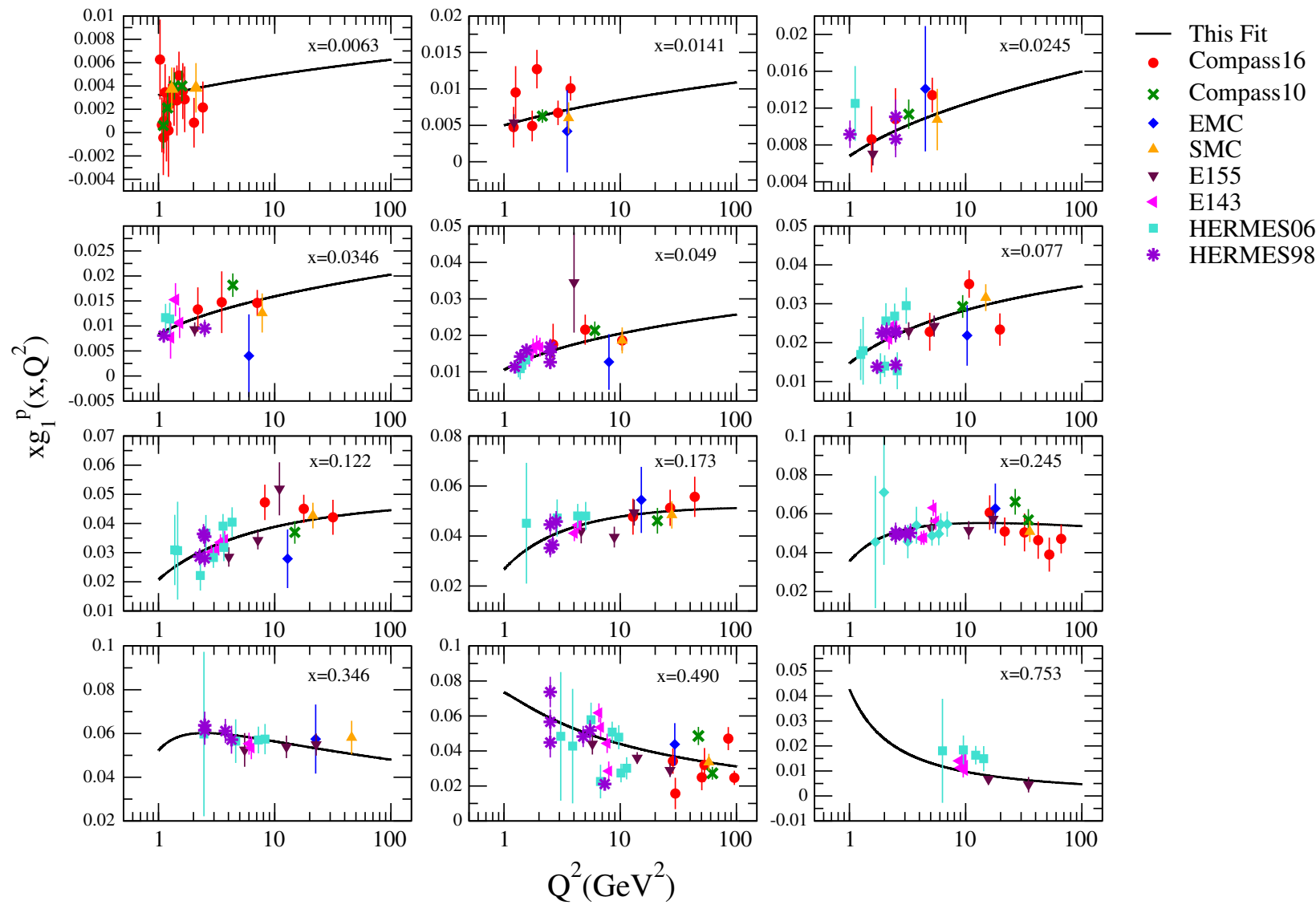

FIG. 2. Polarized proton structure function $x g_{1}^{p}\left(x, Q^{2}\right)$ as a function of $Q^{2}$ in intervals of $x$ in comparison to the experimental data of COMPASS16 [68], COMPASS10 [63], EMC [61], SMC [62], E155 [60], E143 [57], HERMES06 [51], and HERMES98 [52,53]. 
As outlined in Sec. III A, we have a total of nine unknown free parameters: eight parameters describing the PPDFs at $Q_{0}^{2}$, and also $\alpha_{s}\left(Q_{0}^{2}\right)$ as another free parameter. We will use the CERN library MINUIT package [86] to minimize $\chi^{2}$ by varying the free parameters to obtain a best fit. We are now ready to extract the polarized parton densities.

\section{RESULTS OF THE QCD ANALYSIS}

In this section, we will demonstrate how inclusion of the new COMPASS proton $g_{1}^{p}$ data [68] and deuteron $g_{1}^{d}$ data [69] influences our PPDFs.

\section{A. Analysis outline}

\section{The fits: Base, fit $A$, and fit $B$}

We will divide our analysis into three steps. As a first step, we perform a fit with all the data of Table I, with the exception of the COMPASS16 [68] and COMPASS17 [69] experimental data; this totals 379 data points, and we identify this as our "base" fit. We then include the COMPASS16 proton data, and this is our "fit A," which contains 430 data points. Finally, we include the COMPASS17 deuteron data, and this is our "fit B" with the full 473 data points. As fit B contains the complete data set, we will use this for comparisons in Figs. 2-5, where it is identified as "This Fit."

In Table II, the final values of the fit parameters for the different data sets are summarized. We find that $\chi^{2} /$ d.o.f. is less than unity in all cases, indicating a good quality of fit. Additionally, our fits compare well with our previous KATAO analysis, where we find $\chi^{2} /$ d.o.f. $=273.6 / 370$.

\section{B. Structure functions and PPDFs}

\section{The $x g_{1}^{N}$ structure functions vs $Q^{2}$}

We will begin with the comparison of the $x g_{1}^{N}$ structure functions, as this is the primary input to our fit. In Figs. 2-4, we display the comparison of our theoretical predictions with the structure function data for $x g_{1}^{p}, x g_{1}^{d}$, and $x g_{1}^{n}$, respectively. The figures are given as functions of $Q^{2}$ at different values of $x$ and are compared to all of the experimental data that we used in the present analysis. The theoretical predictions are in good agreement with the experimental measurements across the fill $x$ range. In the following sections, we will investigate the impact of the new COMPASS measurements on the central values of the PPDFs and their uncertainties.

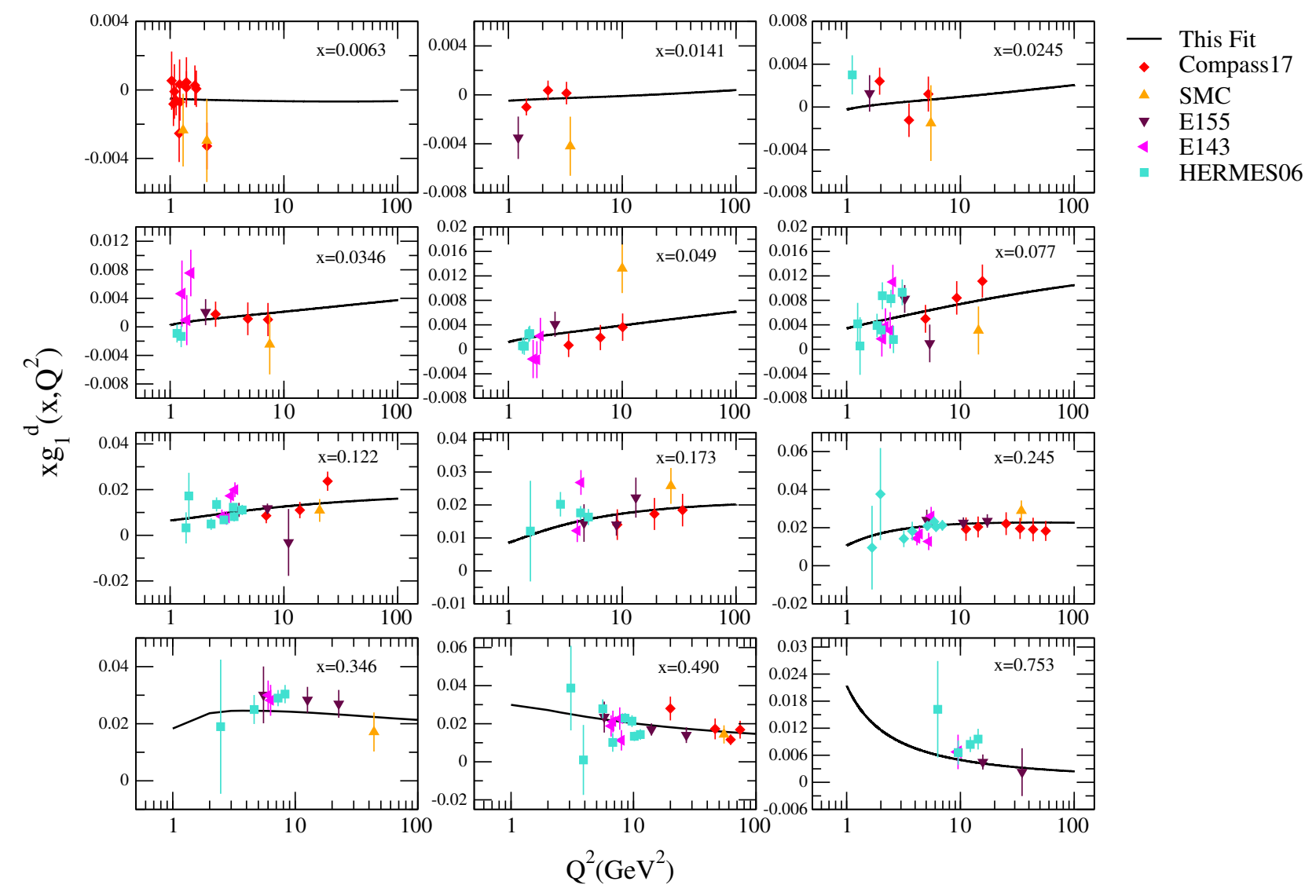

FIG. 3. Polarized deuteron structure function $x g_{1}^{d}\left(x, Q^{2}\right)$ as a function of $Q^{2}$ in intervals of $x$ in comparison to the experimental data of COMPASS17 [69], SMC [62], E155 [58,59], E143 [57], and HERMES06 [51]. 


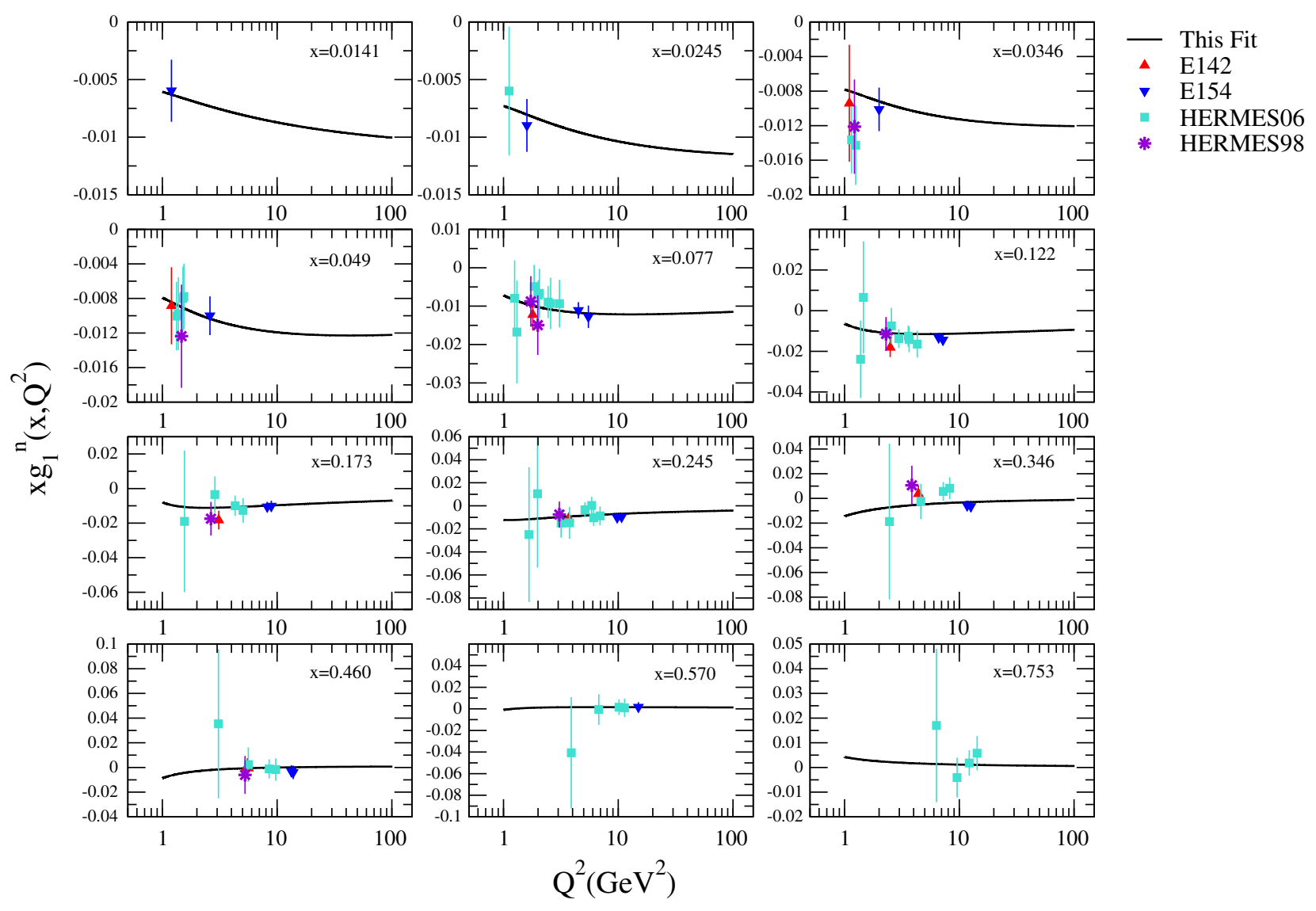

FIG. 4. Polarized neutron structure function $x g_{1}^{n}\left(x, Q^{2}\right)$ as a function of $Q^{2}$ in intervals of $x$ in comparison to the experimental data of E142 [54], E154 [56], HERMES06 [51], and HERMES98 [52,53].

\section{The polarized PDFs (PPDFs)}

Next, we turn to the PPDFs themselves. Figure 5 displays the extracted $x(\delta u+\delta \bar{u})(x), x(\delta d+\delta \bar{d})(x), x(\delta s+\delta \bar{s})(x)$, and $x \delta g(x)$ PPDFs with their associated uncertainties, as compared with various other determinations from the literature $[13,27,31,35,37,44]$.

We derive the uncertainties of the polarized parton distributions for the different polarized observables using the covariance matrix elements of the QCD fit.

Examining Fig. 5, we find that the spread of results for the $x(\delta u+\delta \bar{u})$ distribution is comparatively narrow, indicating that this flavor component is well constrained. The results of "fit B" are comparable to our previous analysis using the Jacobi polynomial expansion method (KATAO) [44], as well as many of the other results from the literature. Our results are slightly larger than those of BB in the larger $x$ region $(x \sim 0.2)$. The $x(\delta d+\delta \bar{d})$ distribution is also comparatively narrow, suggesting this too is well constrained. Again, our results of "fit B" are generally comparable to the other results from the literature, with "fit B" yielding a slightly larger $x(\delta d+\delta \bar{d})$ than BB in the region $x \sim 0.1$. For the $x(\delta s+\delta \bar{s})$ distributions (or $2 \delta \bar{q}$ in our notation), we find a broader spread of both our results ("fit B" and KATAO) and the other fits from the literature, suggesting this component is less constrained. Specifically, "fit B" roughly coincides with many of the other predictions, but the DSSV and LSS10 results yield a change in sign as a function of $x$, and LSS14 yields a larger result. Of all the components we examine, clearly the gluon distribution $x \delta g$ has the widest spread of predictions and the greatest uncertainty. "Fit B" is similar to the KATAO results, but it yields a smaller result in the region $x \sim 0.3$; compared to the other curves, these results generally give a smaller $x \delta g$ than the other predictions. In particular, in the region $x \sim 0.1 \mathrm{AAC}$ gives the largest result and DSSV gives negative results. Clearly, the $x \delta g$ distribution leaves much room for improvement, and it will be interesting to see which predictions are favored by future data sets. Presumably, the choice of data sets (such as SIDIS) may contribute to these differences.

\section{Comparison of \{base, fit A, fit B\} on PPDFs}

Since it is the new COMPASS data on $x g_{1}^{p}$ and $x g_{1}^{d}$ that represent the important new additions to our data set, we want to focus on the variations among our fits: $\{$ base, fit A, fit B .

To investigate the specific impact of COMPASS16 and COMPASS17 data sets, we compare our results for our 

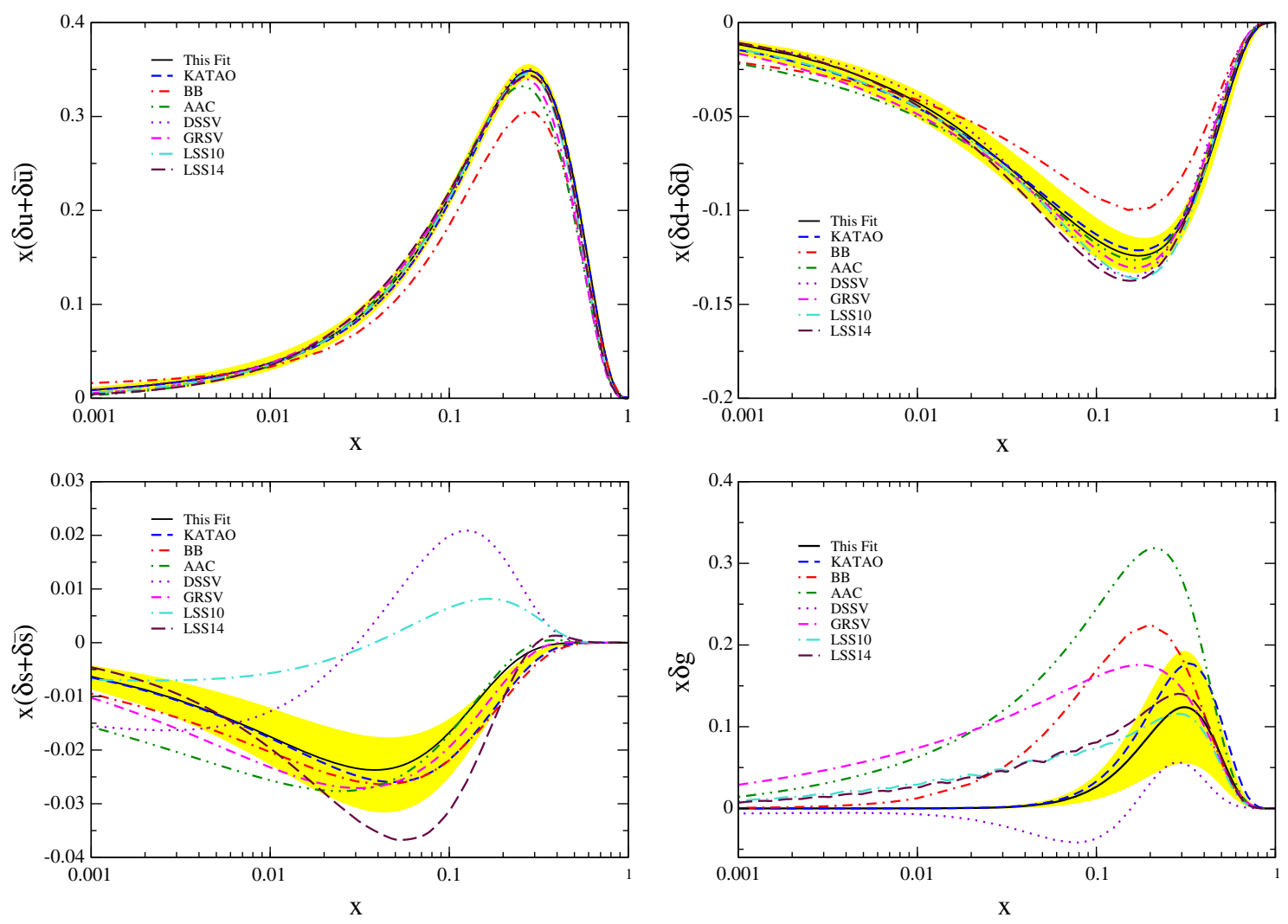

FIG. 5. Our results for NLO polarized parton distributions at $Q_{0}{ }^{2}=4 \mathrm{GeV}^{2}$. The corresponding PPDFs (the solid lines) are shown with error bands compared to results obtained by KATAO [44], BB [37], AAC [31], DSSV [35], GRSV [27], and LSS [13,14]. For clarity, we only present our fit B results, labeled here as "This Fit."

individual fits: "base" (without including COMPASS16 and COMPASS17), "fit A" (including COMPASS16), and "fit B" (including COMPASS16 and COMPASS17). These results are shown in Fig. 6, where we have displayed both the absolute value of the PPDFs and also the ratio compared to our base fit.

As suggested by the results of Fig. 5, in Fig. 6 we find that $x \delta u_{v}(x)$ and $x \delta d_{v}(x)$ appear to be strongly constrained with little variation among the separate fits. Specifically, the variation is on the order of one percent, except for the region at large $x$ where the PPDFs vanish and there are no data constraints.

In contrast, $x \delta \bar{q}(x)$ and $x \delta g(x)$ do display some differences among the fits due to the addition of the COMPASS data; the variations of "fit A" and "fit B" in Fig. 6 are quite similar, and these differ from the "base" fit. The $x \delta \bar{q}(x)$ function displays some variation in the small- $x$ region $\lesssim 10^{-1}$, while the variation of the $x \delta g(x)$ function is generally at larger $x \gtrsim 10^{-1}$; again, the very-large- $x$ region should be discounted as before.

\section{COMPASS $x g_{1}^{N}$ structure functions vs $x$}

To examine how the fits change with the inclusion of the COMPASS data, we examine the partial $\chi^{2}$ contributions to the COMPASS16 and COMPASS17 data set for each of our fits: $\{$ base, fit A, fit B $\}$. If we compute $\chi^{2}$ for the COMPASS16 data set using the "base" fit (which does not include this data), we find a total $\chi^{2}$ value of 34.67 for the 51 COMPASS16 data points, and when we include this data in the fit ("fit A"), it improves slightly to 33.48 . Correspondingly, if we fit the COMPASS17 data set using "fit A" (which does not include this data), we find a total $\chi^{2}$ value of 27.43 for the 43 COMPASS17 data points, and in "fit B," this is quite similar at 27.22. Thus, both the COMPASS16 and COMPASS17 data sets are in reasonable agreement with the initial "base" fit. The changes among the $\{$ base, fit A, fit B $\}$ sets are most evident in the ratio plots of Fig. 6.

Finally, in Figs. 7 and 8, we directly compare our "fit B" with the proton and deuteron polarized structure functions from COMPASS16 [68] and COMPASS17 [69] experimental data in a composite plot; as the individual data range over $Q^{2}$, we display our predictions with selected values of $Q^{2}$ to illustrate the evolution effects. This allows us to see the comparison of data and theory in a compact, albeit approximate, manner.

\section{5. $\alpha_{s}\left(Q^{2}\right)$ comparisons}

In our present fits, we allowed $\alpha_{s}\left(Q_{0}^{2}\right)$ to be a parameter of the fit; these results are summarized in Table II. 
TABLE II. Comparison of the parameter values and their statistical errors at the input scale $Q_{0}^{2}=4 \mathrm{GeV}^{2}$ in the different cases: KATAO (Jacobi polynomial method) [44], Base (without COMPASS16 and COMPASS17), Fit A (with COMPASS16), and Fit B (with COMPASS16 and COMPASS17) obtained from the best fit to the data.

\begin{tabular}{|c|c|c|c|c|}
\hline & KATAO (Jacobi Poly.) & Base & Fit A & Fit B \\
\hline$\eta_{u_{v}}$ & 0.928 (fixed) & 0.928 (fixed) & 0.928 (fixed) & 0.928 (fixed) \\
\hline$\alpha_{u_{v}}$ & $0.535 \pm 0.022$ & $0.574 \pm 0.022$ & $0.562 \pm 0.020$ & $0.570 \pm 0.019$ \\
\hline$\beta_{u_{v}}^{u_{v}}$ & $3.222 \pm 0.085$ & $3.208 \pm 0.087$ & $3.187 \pm 0.082$ & $3.207 \pm 0.079$ \\
\hline$\gamma_{u_{v}}$ & 8.180 (fixed) & 6.527 (fixed) & 6.527 (fixed) & 6.527 (fixed) \\
\hline$\eta_{d_{v}}$ & -0.342 (fixed) & -0.342 (fixed) & -0.342 (fixed) & -0.342 (fixed) \\
\hline$\alpha_{d_{v}}$ & $0.530 \pm 0.067$ & $0.561 \pm 0.066$ & $0.591 \pm 0.063$ & $0.606 \pm 0.060$ \\
\hline$\beta_{d_{v}}$ & $3.878 \pm 0.451$ & $3.707 \pm 0.417$ & $3.895 \pm 0.415$ & $3.917 \pm 0.401$ \\
\hline$\gamma_{d_{v}}$ & 4.789 (fixed) & 3.537 (fixed) & 3.537 (fixed) & 3.537 (fixed) \\
\hline$\eta_{\bar{q}}$ & $-0.054 \pm 0.029$ & $-0.328 \pm 0.031$ & $-0.337 \pm 0.035$ & $-0.309 \pm 0.018$ \\
\hline$\alpha_{\bar{q}}$ & $0.474 \pm 0.121$ & $0.500 \pm 0.125$ & $0.421 \pm 0.105$ & $0.474 \pm 0.090$ \\
\hline$\beta_{\bar{q}}^{q}$ & 9.310 (fixed) & 10.243 (fixed) & 10.243 (fixed) & 10.243 (fixed) \\
\hline$\gamma_{\bar{q}}$ & 0 & 0 & 0 & 0 \\
\hline$\eta_{g}$ & $0.224 \pm 0.118$ & $0.231 \pm 0.102$ & $0.161 \pm 0.092$ & $0.158 \pm 0.084$ \\
\hline$\alpha_{g}$ & $2.833 \pm 0.528$ & $2.737 \pm 0.456$ & $2.531 \pm 1.441$ & $2.848 \pm 0.494$ \\
\hline$\beta_{g}$ & 5.747 (fixed) & 6.323 (fixed) & 6.323 (fixed) & 6.323 (fixed) \\
\hline$\gamma_{g}$ & 0 & 0 & 0 & 0 \\
\hline$\alpha_{s}\left(Q_{0}^{2}\right)$ & $0.381 \pm 0.017$ & $0.385 \pm 0.016$ & $0.388 \pm 0.015$ & $0.392 \pm 0.014$ \\
\hline$\chi_{\text {COMPASS } 16}^{2}$ & $\cdots$ & $\ldots$ & 32.732 & 33.032 \\
\hline$\chi_{\text {COMPASS } 17}^{2}$ & $\ldots$ & $\ldots$ & $\ldots$ & 28.074 \\
\hline$\chi^{2}$ & 273.6 & 274.8 & 308.2 & 337.6 \\
\hline d.o.f. & 370 & 370 & 421 & 464 \\
\hline$\chi^{2} /$ d.o.f. & 0.74 & 0.74 & 0.73 & 0.73 \\
\hline
\end{tabular}

We observe that the variation across our different fits is minimal, and these values are consistent with the KATAO fit within uncertainties. Although these values are extracted from data in the range $1 \lesssim Q^{2} \lesssim 100$, we can evolve these up to $M_{Z}$ to compare with other values used in the literature. Note that the $\alpha_{s}\left(Q^{2}\right)$ evolution up to the $M_{Z}^{2}$ scale will depend on the number of active flavors and the mass scale of the transitions; we choose $m_{c}^{2}=3 \mathrm{GeV}^{2}$ and $m_{b}^{2}=25 \mathrm{GeV}^{2}$. Extrapolating our results up to $M_{Z}$ at NLO, we find $\alpha_{s}\left(M_{Z}^{2}\right)=0.1155$ for fit A and $\alpha_{s}\left(M_{Z}^{2}\right)=0.1158$ for fit $\mathrm{B}$, and $\alpha_{s}\left(M_{Z}^{2}\right)=0.1149$ for KATAO. These values are low but within $2 \sigma$ as compared to the world average value of $\alpha_{s}\left(M_{Z}^{2}\right)=0.1181 \pm 0.0011$ [87], and we display this in Fig. 9 along with various results from the literature.

\section{Moments and sum rules}

We now turn to integrated moments and sum rules. Note that the calculation of the moments integrates over the full range $x=[0,1]$, so this requires some extrapolation outside the $x$ range where the structure functions have been measured.

\section{PPDF moments}

We start by computing the PPDF moments, as these will be the necessary ingredients for the other moments and sum rules that follow.
In Table III, we compare the results of the first moments of the polarized parton densities for our fits with results from the literature at NLO in the $\overline{\mathrm{MS}}$ scheme at $Q^{2}=4 \mathrm{GeV}^{2}$. Comparing our "base" fit with "fit A" and "fit B," we see the moments are generally stable, with the exception of $\Delta g$, which varies by $\sim 30 \%$. Including the other PPDF moments from the literature, we see that the results for $\left\{\Delta u_{v}, \Delta d_{v}\right\}$ are quite stable $(\sim 1 \%)$, while $\{\Delta u, \Delta d\}$ show a bit more variation $(\sim 10 \%)$, and finally $\{\Delta \bar{q}, \Delta g\}$ a larger spread (>100\%). We will now look at the influence of the above PPDF moments on the experimentally measurable structure functions.

\section{Structure function moments $\Gamma_{1}^{N}\left(Q^{2}\right)$}

We next examine the first moment of the $x g_{1}^{N}(N=p, d$, n) structure functions, defined to be

$$
\Gamma_{1}^{N}\left(Q^{2}\right) \equiv \int_{0}^{1} g_{1}^{N}\left(x, Q^{2}\right) d x
$$

In Table IV, we compare the results for $\Gamma_{1}^{N}\left(Q^{2}\right)$ of fit B with the COMPASS measurements. We observe that the fit agrees with the COMPASS results within $\sim 1 \sigma$ of the experimental uncertainty.

Next, in Table V, we compare our first moment results with those from the literature. The theoretical results for $\Gamma_{1}^{p}$ 

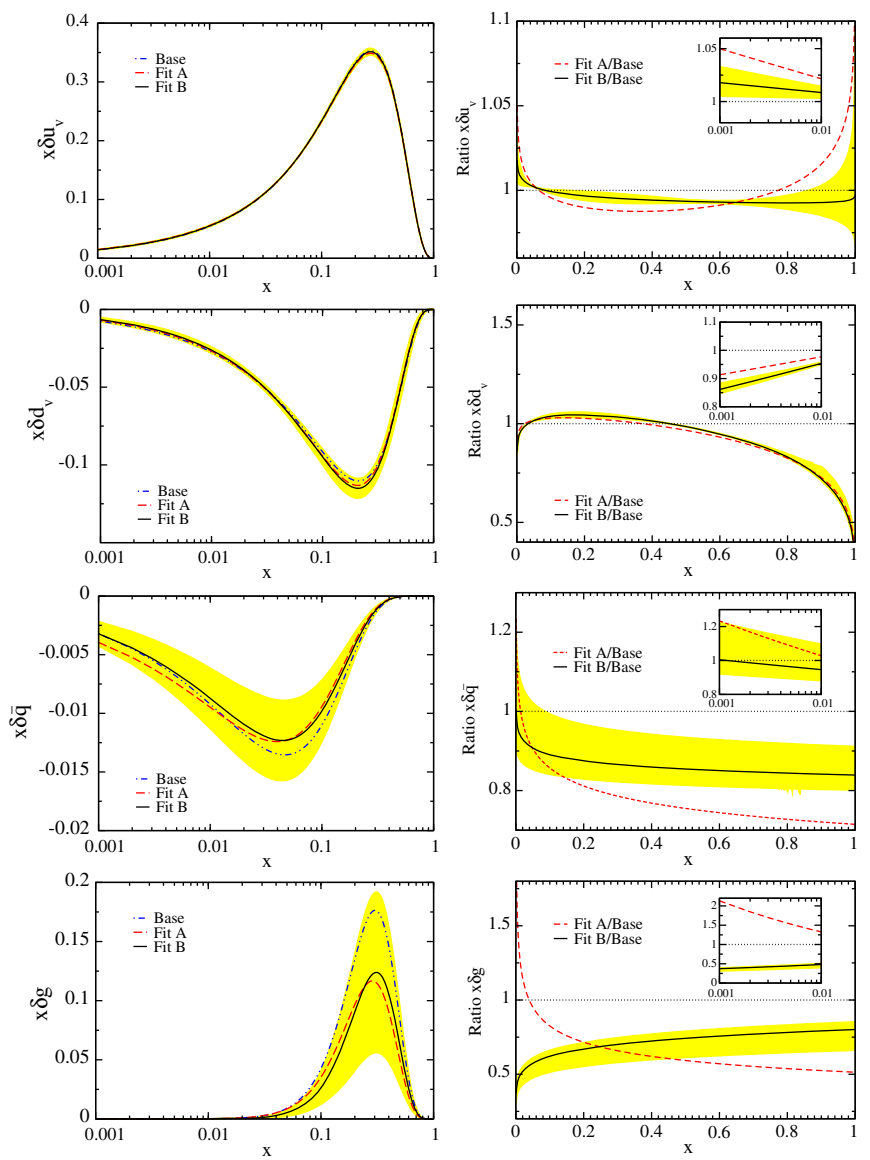

FIG. 6. Left panels: Polarized parton distributions at $Q_{0}^{2}$ as a function of $x$ for our different cases: base (dash-dot-dotted), fit A (dashed), and fit B (solid line) according to Table I. Only the PPDF error bands for fit B (all data) are shown. Right panels: Polarized parton distribution ratios $\delta f / \delta f_{\text {Base }}$ for fit A (dashed) and fit B (solid line) to base, obtained from our QCD fits to the data. The impact of COMPASS data in the low- $x$ regions is shown in the inset plots.

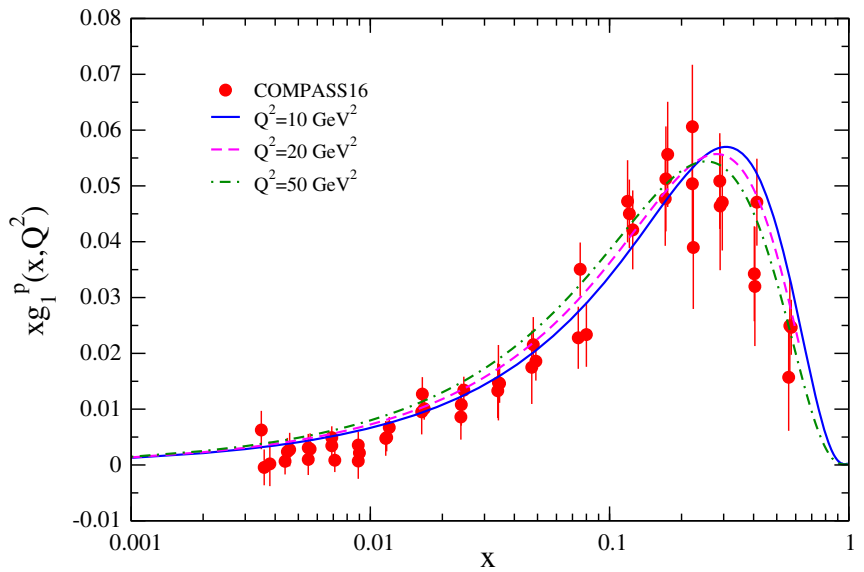

FIG. 7. The COMPASS16 [68] data for the proton structure function $x g_{1}^{p}\left(x, Q^{2}\right)$ compared with our NLO results calculated at $Q^{2}=10,20,50 \mathrm{GeV}^{2}$.

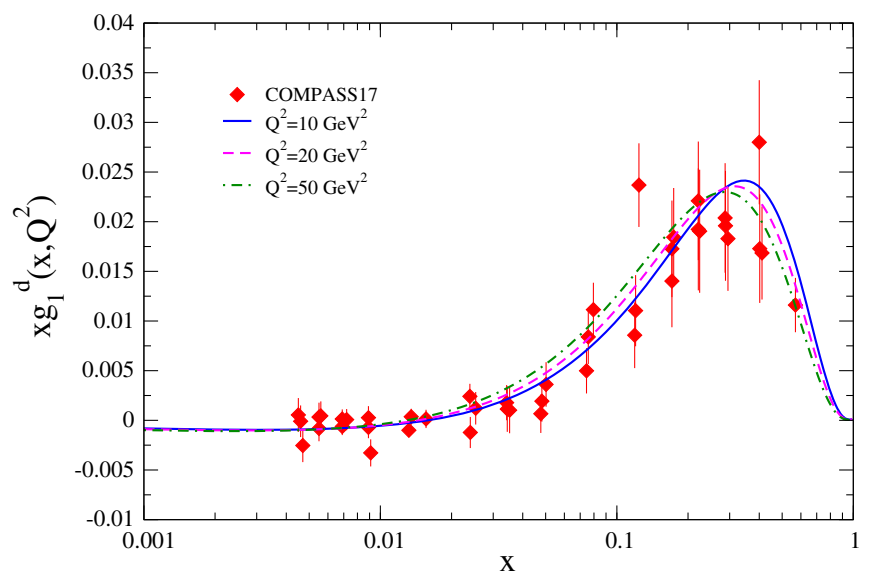

FIG. 8. The COMPASS17 [69] data for the deuteron structure function $x g_{1}^{d}\left(x, Q^{2}\right)$ compared with our NLO results ("fit B") calculated at $Q^{2}=10,20,50 \mathrm{GeV}^{2}$.

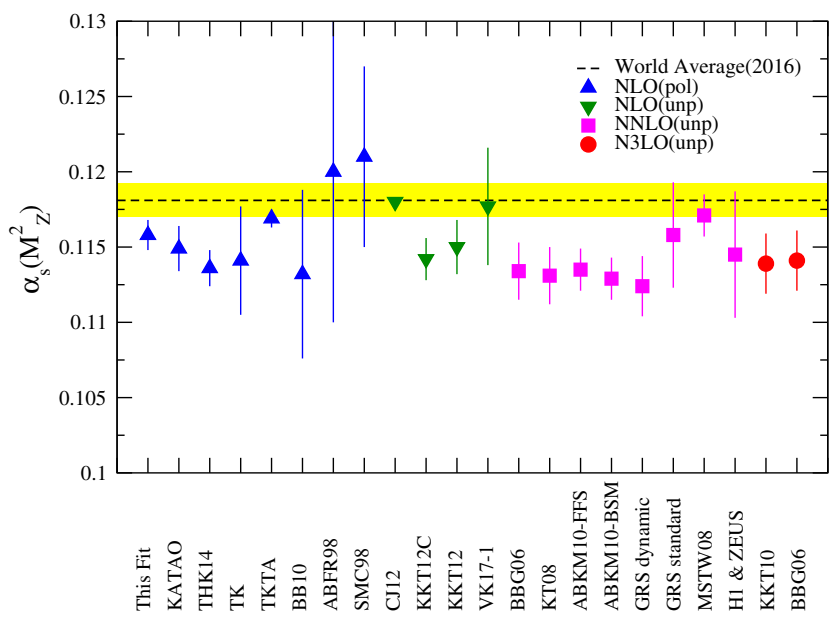

FIG. 9. The strong coupling constant $\alpha_{s}\left(M_{Z}^{2}\right)$ values as compared with different QCD analyses at NLO [16,26,37,44,45, 48,88-91], NNLO [92-98], and NNNLO [92,99]. The dashed line and yellow band shows the world average, $\alpha_{s}\left(M_{Z}^{2}\right)=$ $0.1181 \pm 0.0011$ [87].

TABLE III. Comparison of the first moments of the polarized parton densities at NLO in the $\overline{\mathrm{MS}}$ scheme at $Q^{2}=4 \mathrm{GeV}^{2}$.

\begin{tabular}{lrrrrrrr}
\hline \hline & & & & KATAO & BB & GRSV & AAC \\
& \multicolumn{1}{c}{ Base } & Fit A & Fit B & {$[44]$} & {$[37]$} & {$[27]$} & {$[31]$} \\
\hline$\Delta u_{v}$ & 0.928 & 0.928 & 0.928 & 0.928 & 0.928 & 0.9206 & 0.9278 \\
$\Delta d_{v}$ & -0.342 & -0.342 & -0.342 & -0.342 & -0.342 & -0.3409 & -0.3416 \\
$\Delta u$ & 0.873 & 0.872 & 0.876 & 0.874 & 0.866 & 0.8593 & 0.8399 \\
$\Delta d$ & -0.397 & -0.398 & -0.394 & -0.396 & -0.404 & -0.4043 & -0.4295 \\
$\Delta \bar{q}$ & -0.055 & -0.056 & -0.052 & -0.054 & -0.066 & -0.0625 & -0.0879 \\
$\Delta g$ & 0.231 & 0.161 & 0.158 & 0.224 & 0.462 & 0.6828 & 0.8076 \\
\hline \hline
\end{tabular}


TABLE IV. First moments of the polarized structure function $\left\{\Gamma_{1}^{p}, \Gamma_{1}^{d}, \Gamma_{1}^{n}, \Gamma_{1}^{\mathrm{NS}}\right\}$ for "fit B" at NLO at $\mathrm{Q}^{2}=3 \mathrm{GeV}^{2}$ compared with COMPASS16 [68] and COMPASS17 [69] experimental data.

\begin{tabular}{lrcc}
\hline \hline & Fit B & COMPASS16 [68] & COMPASS17 [69] \\
\hline$\Gamma_{1}^{p}$ & 0.133 & $0.139 \pm 0.003 \pm 0.009$ & $\ldots$ \\
$\Gamma_{1}^{d}$ & 0.040 & $\ldots$ & $0.043 \pm 0.001 \pm 0.003$ \\
$\Gamma_{1}^{n}$ & -0.048 & $-0.041 \pm 0.006 \pm 0.011$ & $\ldots$ \\
$\Gamma_{1}^{\mathrm{NS}}$ & 0.182 & $0.181 \pm 0.008 \pm 0.014$ & $0.192 \pm 0.007 \pm 0.015$ \\
\hline \hline
\end{tabular}

TABLE V. First moments of the polarized structure functions $\left\{\Gamma_{1}^{p}, \Gamma_{1}^{d}, \Gamma_{1}^{n}\right\}$ at $Q^{2}=5 \mathrm{GeV}^{2}$ for "fit B" as compared to other results from the literature at NLO in the $\overline{\mathrm{MS}}$ scheme.

\begin{tabular}{ccccc}
\hline \hline & Fit B & KATAO [44] & GRSV [27] & AAC [31] \\
\hline$\Gamma_{1}^{p}$ & 0.135 & 0.133 & 0.132 & 0.137 \\
$\Gamma_{1}^{d}$ & 0.041 & 0.036 & 0.032 & 0.038 \\
$\Gamma_{1}^{n}$ & -0.045 & -0.053 & -0.062 & -0.056 \\
\hline \hline
\end{tabular}
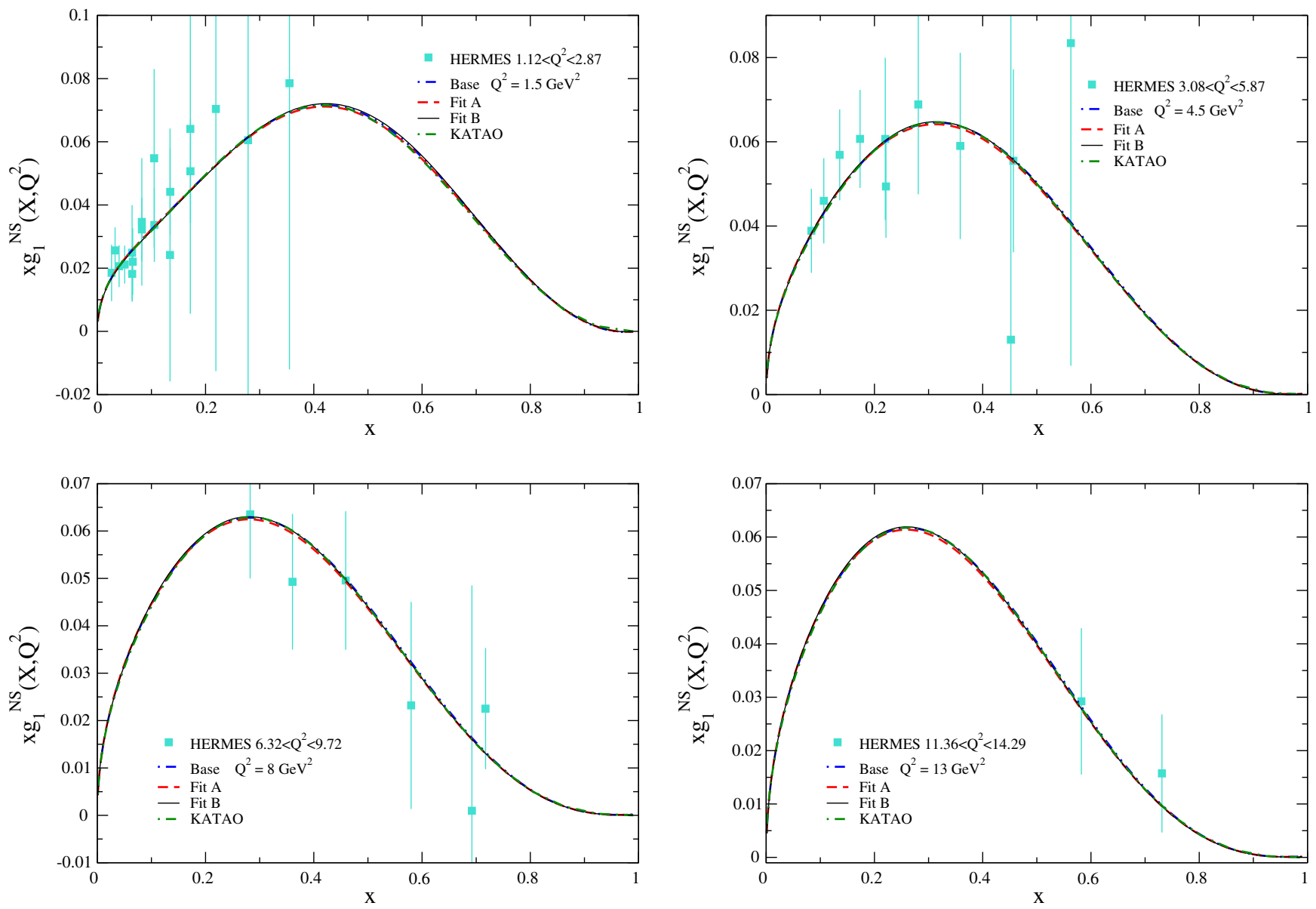

FIG. 10. NLO nonsinglet polarized structure function $x g_{1}^{\mathrm{NS}}\left(x, Q^{2}\right)$ as a function of $x$ in comparison with the results of KATAO [44] and HERMES experimental data [51]. 
$\Gamma_{1}^{\mathrm{NS}}\left(Q^{2}\right)$ enters the polarized Bjorken sum rule [100] and is related to the ratio of the axial and vector coupling constants $\left(g_{A, V}\right)$. Here, $C_{1}^{\mathrm{NS}}\left(Q^{2}\right)$ is the nonsinglet coefficient function.

In a similar manner, we define $g_{1}^{\mathrm{NS}}\left(x, Q^{2}\right)$ as the difference between the proton and neutron structure functions:

$$
\begin{aligned}
x g_{1}^{\mathrm{NS}}\left(x, Q^{2}\right) & \equiv x g_{1}^{p}\left(x, Q^{2}\right)-x g_{1}^{n}\left(x, Q^{2}\right) \\
& =2\left[x g_{1}^{p}\left(x, Q^{2}\right)-\frac{x g_{1}^{d}\left(x, Q^{2}\right)}{1-\frac{3}{2} \omega_{D}}\right] .
\end{aligned}
$$

In Fig. 10, we compare our results for $x g_{1}^{\mathrm{NS}}\left(x, Q^{2}\right)$ with the HERMES experimental data [51] for selected bins of $Q^{2}$. We find minimal variation among our different theoretical fits (including the previous KATAO fit), and these curves compare well with the experimental results.

From Eq. (20), we can also relate $\Gamma_{1}^{\mathrm{NS}}\left(Q^{2}\right)$ to the previously computed proton and neutron first moments as

$$
\Gamma_{1}^{\mathrm{NS}}\left(Q^{2}\right)=\Gamma_{1}^{p}\left(Q^{2}\right)-\Gamma_{1}^{n}\left(Q^{2}\right) .
$$

These results are presented in Table $\mathrm{V}$ and with the COMPASS results. The result of our "fit B" is comparable to COMPASS16, and below (but within uncertainties to) COMPASS17.

\section{4. $g_{2}^{N}\left(x, Q^{2}\right)$ structure functions}

We can also calculate the structure function $g_{2}^{N}\left(x, Q^{2}\right)$ via the Wandzura-Wilczek relation $[101,102]$ :

$$
g_{2}^{N}\left(x, Q^{2}\right)=-g_{1}^{N}\left(x, Q^{2}\right)+\int_{x}^{1} \frac{d y}{y} g_{1}^{N}\left(y, Q^{2}\right) .
$$

Figure 11 shows the polarized structure function $x g_{2}^{p}$ and $x g_{2}^{d}$ as a function of $x$ for different cases of base, fit A, fit B, and our previous KATAO results [44] in comparison with E143 [57], E155 [103], HERMES [104], and SMC [105] experimental data at $Q^{2}=5,6 \mathrm{GeV}^{2}$. As the data actually span over a range of $Q^{2}$, in Fig. 12 we display the $Q^{2}$ evolution of the polarized structure function $x g_{2}\left(x, Q^{2}\right)$ for the proton and deuteron as a function of $x$. In Fig. 11 we see that our "base" and "fit A" coincide throughout the $x$ range, suggesting a minimal impact from the COMPASS16 data on this observable; conversely, our "fit B" does differ, especially in the larger- $x$ region, suggesting a stronger influence of the COMPASS17 data on $x g_{2}^{d}\left(x, Q^{2}\right)$.

\section{The proton spin}

It is important for us to understand the decomposition of the proton spin in terms of the separate contributions from the quarks, gluon, and the orbital angular momentum components. The spin of the proton can be computed from the first moment of the polarized parton densities together
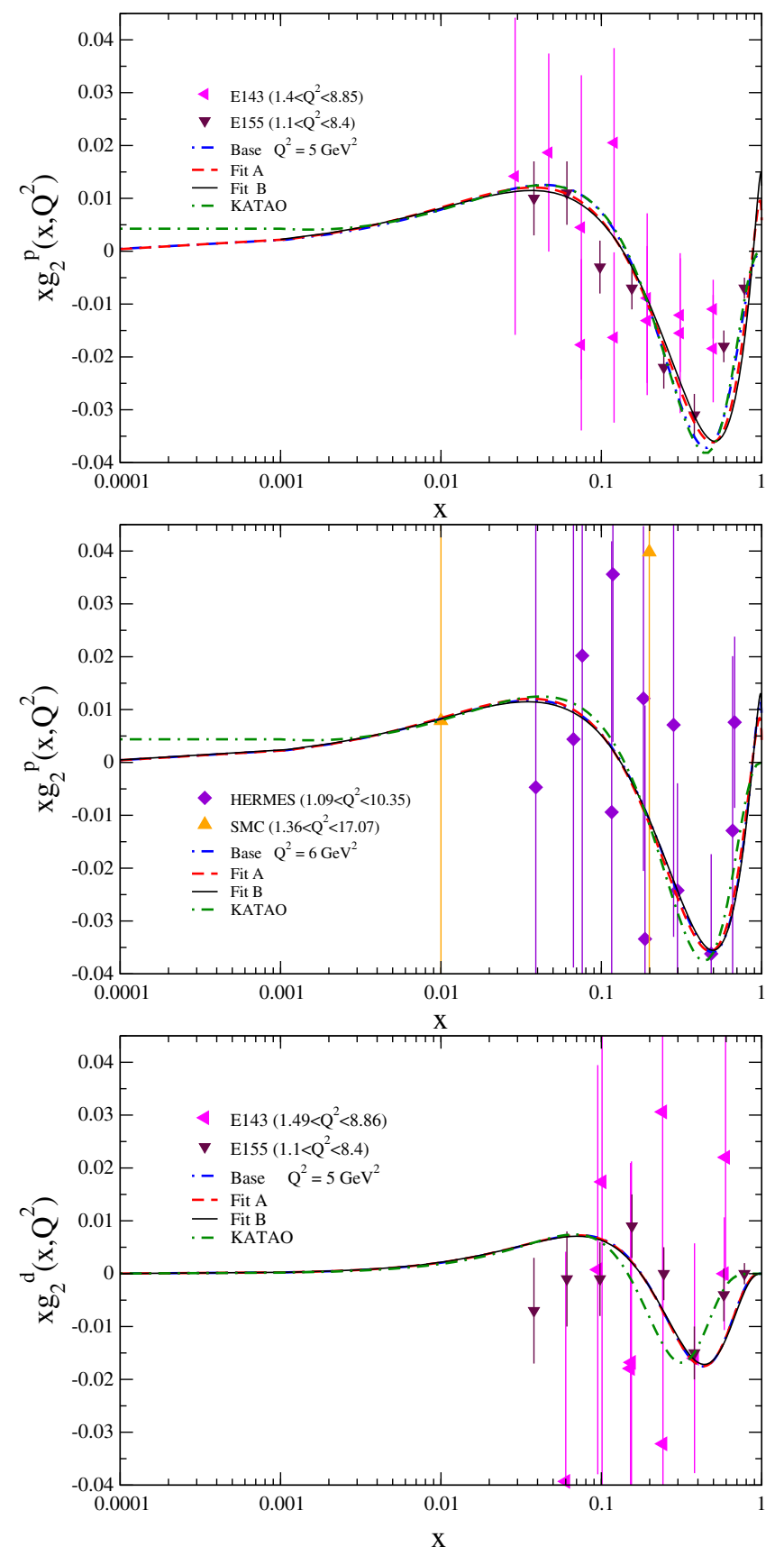

FIG. 11. NLO polarized structure function $x g_{2}^{p}\left(x, Q^{2}\right)$ and $x g_{2}^{d}\left(x, Q^{2}\right)$ as a function of $x$ for $Q^{2}=5,6 \mathrm{GeV}^{2}$ compared to E143 [57], E155 [103], HERMES [104], and SMC [105] experimental data. We present also the results of different base (dash-dot-dotted), fit A (dashed), and fit B (solid line) QCD fits, which are compared with our previous KATAO (dash-dotted) results.

with the quark and gluon orbital momentum $\left(L_{q}, L_{g}\right)$ as follows [106]:

$$
\frac{1}{2}=\frac{1}{2} \Delta \Sigma\left(Q^{2}\right)+\Delta g\left(Q^{2}\right)+\mathrm{L}_{z}\left(Q^{2}\right) .
$$



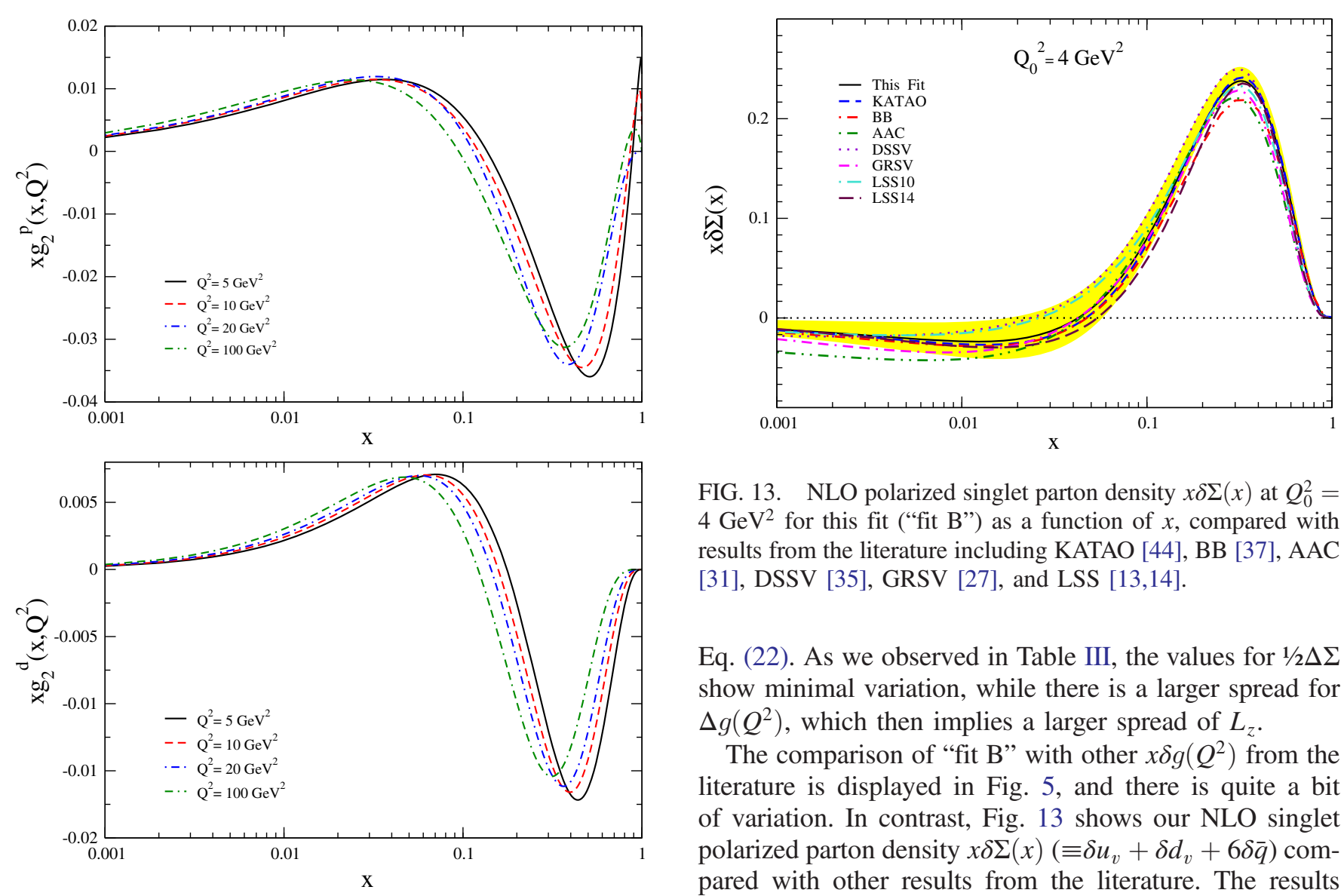

FIG. 13. NLO polarized singlet parton density $x \delta \Sigma(x)$ at $Q_{0}^{2}=$ $4 \mathrm{GeV}^{2}$ for this fit ("fit B") as a function of $x$, compared with results from the literature including KATAO [44], BB [37], AAC [31], DSSV [35], GRSV [27], and LSS [13,14].

Eq. (22). As we observed in Table III, the values for $1 / 2 \Delta \Sigma$ show minimal variation, while there is a larger spread for $\Delta g\left(Q^{2}\right)$, which then implies a larger spread of $L_{z}$.

The comparison of "fit B" with other $x \delta g\left(Q^{2}\right)$ from the literature is displayed in Fig. 5, and there is quite a bit of variation. In contrast, Fig. 13 shows our NLO singlet polarized parton density $x \delta \Sigma(x)\left(\equiv \delta u_{v}+\delta d_{v}+6 \delta \bar{q}\right)$ compared with other results from the literature. The results of this fit ("fit B") with the previous KATAO analysis [44]

FIG. 12. NLO polarized structure function $x g_{2}\left(x, Q^{2}\right)$ for the proton and deuteron as function of $x$ and for $Q^{2}=5,10,20$, $100 \mathrm{GeV}^{2}$.

Here $\mathrm{L}_{z}\left(Q^{2}\right)=\mathrm{L}_{q}\left(Q^{2}\right)+\mathrm{L}_{g}\left(Q^{2}\right)$ is the total orbital angular momentum of all the quarks and gluons, $\Delta g\left(Q^{2}\right)=$ $\int_{0}^{1} d x \delta g\left(x, Q^{2}\right)$ is the first moment of the polarized gluon distribution, and $\Delta \Sigma\left(Q^{2}\right)=\int_{0}^{1} d x \delta \Sigma\left(x, Q^{2}\right)$ with $\delta \Sigma \equiv$ $\delta u_{v}+\delta d_{v}+6 \delta \bar{q}$ is the first moment of the polarized singlet distribution. In Eq. (22), we note that the spin sum (1/2) is actually independent of $Q^{2}$ even though each individual term is dependent on $Q^{2}$.

In Table VI, we compute $\{1 / 2 \Delta \Sigma, \Delta g\}$ using "fit B" at $Q^{2}=4 \mathrm{GeV}^{2}$, and then infer the value of $\mathrm{L}_{z}\left(Q^{2}\right)$ assuming

TABLE VI. Spin contribution of the proton in the NLO approximations at $Q^{2}=4 \mathrm{GeV}^{2}$ for base, fit A, and fit $\mathrm{B}$ compared with the KATAO data [44]. We have computed $\left\{\frac{1}{2} \Delta \Sigma, \Delta g\right\}$ using our PPDFs and inferred $L_{z}$ assuming precisely $1 / 2$ for the proton spin.

\begin{tabular}{lcccc}
\hline \hline & $\frac{1}{2} \Delta \Sigma$ & $\Delta g$ & $L_{z}$ & $\frac{1}{2} \Delta \Sigma+\Delta g+L_{z}$ \\
\hline KATAO & 0.131 & 0.224 & 0.145 & $1 / 2$ \\
Base & 0.129 & 0.231 & 0.140 & $1 / 2$ \\
Fit A & 0.125 & 0.161 & 0.214 & $1 / 2$ \\
Fit B & 0.139 & 0.158 & 0.203 & $1 / 2$ \\
\hline \hline
\end{tabular}
are quite similar, as suggested by Table III. Generally, the singlet polarized distributions are negative for $x \lesssim 0.04$ for most of the models, but there is some slight variation in the range $x \lesssim 0.06$ to $x \lesssim 0.02$. Overall, the variation of $x \delta \Sigma(x)$, as compared to $x \delta g\left(Q^{2}\right)$, is reduced; this is notable, as $x \delta \Sigma(x)$ is a combination of both valence and sea PPDFs.

\section{CONCLUSIONS}

We performed a QCD analysis of the deep inelastic nucleon scattering data from COMPASS [63-65], HERMES [51-53], SLAC [54,56-60], EMC [61], and SMC [62] at NLO. This also included the recent data from COMPASS16 [68] and COMPASS17 [69] for the proton and deuteron polarized structure function measurements.

We extracted the PPDFs and $\alpha_{s}\left(Q_{0}^{2}\right)$ with uncertainties using a $\chi^{2}$ minimization and compared our results with those from the literature, including AAC [31], DSSV [35], BB [37], GRSV [27], LSS [13,14], and KATAO [44]. In contrast to our previous polarized analysis (KATAO), we did not use the Jacobi polynomial expansion method. Our results for the PPDFs are comparable to other extractions, and generally it appears that $x \delta u_{v}$ and $x \delta d_{v}$ are comparatively well determined in contrast to $x \delta \bar{q}$ and $x \delta g$, which display a larger variation across the $x$ range.

We also computed various structure functions and moments for the proton, neutron, and deuteron, and these 
also compare well with the COMPASS data, as well as other determinations from the literature. Again, the results from this fit are comparable to the previous KATAO [44] results using orthogonal polynomials; it is reassuring to see that the results are generally independent of the underlying calculational methodology.

The strong coupling constant $\alpha_{s}\left(Q_{0}^{2}\right)$ was extracted from the fits, and the uncertainty is slightly decreased compared to the KATAO analysis. This $\alpha_{s}\left(Q_{0}^{2}\right)$ can be evolved up to $\alpha_{s}\left(M_{Z}^{2}\right)$ by assuming an evolution order (LO, NLO, ...) and heavy quark mass thresholds; we find values that are low compared to the world average, but within uncertainties.

From this analysis, it appears that the various theoretical analyses using a variety of techniques and $x$-space, $N$-space, or orthogonal polynomials are generally converging to yield a homogeneous set of predictions which are in good agreement with the diverse sets of experimental measurements. While there is still room for further improvements, such studies provide a strong validation of the underlying QCD theoretical framework.

A standard LHAPDF library file of our polarized PDFs $\left\{x \delta u_{v}\left(x, Q^{2}\right), x \delta d_{v}\left(x, Q^{2}\right), x \delta \bar{q}\left(x, Q^{2}\right), x \delta g\left(x, Q^{2}\right)\right\}$ and their uncertainties can be obtained via e-mail from the authors upon request.

\section{ACKNOWLEDGMENTS}

We gratefully acknowledge O. Denisov and E. Kabuss of the COMPASS Collaboration, as well as D. Stamenov and S. Bass for detailed comments and helpful discussions and suggestions. We also thank F. Arbabifar and M. Soleymanini for useful comments. A. K. is grateful to the CERN TH-PH division for their hospitality where a portion of this work was performed. The work of F. I. O. was supported in part by the U.S. Department of Energy under Grant No. DE-SC0010129.
[1] S. D. Bass, Rev. Mod. Phys. 77, 1257 (2005).

[2] S. E. Kuhn, J. P. Chen, and E. Leader, Prog. Part. Nucl. Phys. 63, 1 (2009).

[3] M. Anselmino, A. Efremov, and E. Leader, Phys. Rep. 261, 1 (1995); 281, 399(E) (1997).

[4] B. Lampe and E. Reya, Phys. Rep. 332, 1 (2000).

[5] E. W. Hughes and R. Voss, Annu. Rev. Nucl. Part. Sci. 49, 303 (1999).

[6] B. W. Filippone and X.-D. Ji, Adv. Nucl. Phys. 26, 1 (2001).

[7] G. Altarelli, arXiv:0907.1751.

[8] A. V. Sidorov and D. B. Stamenov, Mod. Phys. Lett. A 21, 1991 (2006).

[9] E. Leader, A. V. Sidorov, and D. B. Stamenov, Phys. Rev. D 75, 074027 (2007).

[10] E. Leader, A. V. Sidorov, and D. B. Stamenov, in 11th International Workshop on High Energy Spin Physics (DUBNA-SPIN-05), Dubna, Russia, 2005, pp. 152-163.

[11] E. Leader, A. V. Sidorov, and D. B. Stamenov, Phys. Rev. D 73, 034023 (2006).

[12] E. Leader, A. V. Sidorov, and D. B. Stamenov, J. High Energy Phys. 06 (2005) 033.

[13] E. Leader, A. V. Sidorov, and D. B. Stamenov, Phys. Rev. D 82, 114018 (2010).

[14] E. Leader, A. V. Sidorov, and D. B. Stamenov, Phys. Rev. D 91, 054017 (2015).

[15] E. Leader, A. V. Sidorov, and D. B. Stamenov, Czech. J. Phys. 54, B21 (2004).

[16] G. Altarelli, R. D. Ball, S. Forte, and G. Ridolfi, Acta Phys. Polon. B 29, 1145 (1998).

[17] R. D. Ball, G. Ridolfi, G. Altarelli, and S. Forte, AIP Conf. Proc. 407, 834 (1997).

[18] C. Bourrely, F. Buccella, O. Pisanti, P. Santorelli, and J. Soffer, Prog. Theor. Phys. 99, 1017 (1998).
[19] D. de Florian, O. A. Sampayo, and R. Sassot, Phys. Rev. D 57, 5803 (1998).

[20] D. de Florian and R. Sassot, Phys. Rev. D 62, 094025 (2000).

[21] D. de Florian, G. A. Navarro, and R. Sassot, Phys. Rev. D 71, 094018 (2005).

[22] L. E. Gordon, M. Goshtasbpour, and G. P. Ramsey, Phys. Rev. D 58, 094017 (1998).

[23] E. Leader, A. V. Sidorov, and D. B. Stamenov, Phys. Lett. B 462, 189 (1999).

[24] E. Leader, A. V. Sidorov, and D. B. Stamenov, Phys. Lett. B 445, 232 (1998).

[25] E. Leader, A. V. Sidorov, and D. B. Stamenov, Int. J. Mod. Phys. A 13, 5573 (1998).

[26] D. K. Ghosh, S. Gupta, and D. Indumathi, Phys. Rev. D 62, 094012 (2000).

[27] M. Gluck, E. Reya, M. Stratmann, and W. Vogelsang, Phys. Rev. D 63, 094005 (2001).

[28] R. S. Bhalerao, Phys. Rev. C 63, 025208 (2001).

[29] E. Leader, A. V. Sidorov, and D. B. Stamenov, Eur. Phys. J. C 23, 479 (2002).

[30] J. Blumlein and H. Bottcher, Nucl. Phys. B636, 225 (2002).

[31] Y. Goto et al. (Asymmetry Analysis Collaboration), Phys. Rev. D 62, 034017 (2000).

[32] C. Bourrely, J. Soffer, and F. Buccella, Eur. Phys. J. C 23, 487 (2002).

[33] S. Forte, M. L. Mangano, and G. Ridolfi, Nucl. Phys. B602, 585 (2001).

[34] G. Altarelli, R. D. Ball, S. Forte, and G. Ridolfi, Nucl. Phys. B496, 337 (1997).

[35] D. de Florian, R. Sassot, M. Stratmann, and W. Vogelsang, Phys. Rev. Lett. 101, 072001 (2008).

[36] M. Hirai and S. Kumano (Asymmetry Analysis Collaboration), Nucl. Phys. B813, 106 (2009). 
[37] J. Blumlein and H. Bottcher, Nucl. Phys. B841, 205 (2010).

[38] D. de Florian, R. Sassot, M. Stratmann, and W. Vogelsang, Phys. Rev. D 80, 034030 (2009).

[39] E. Leader, A. V. Sidorov, and D. B. Stamenov, Phys. Rev. D 84, 014002 (2011).

[40] E. Leader, A. V. Sidorov, and D. B. Stamenov, arXiv: 1007.4781.

[41] E. Leader, A. V. Sidorov, and D. B. Stamenov, Phys. Rev. D 80, 054026 (2009).

[42] R. D. Ball, S. Forte, A. Guffanti, E. R. Nocera, G. Ridolfi, and J. Rojo (NNPDF Collaboration), Nucl. Phys. B874, 36 (2013).

[43] E. R. Nocera, Phys. Lett. B 742, 117 (2015).

[44] A. N. Khorramian, S. A. Tehrani, S. Taheri Monfared, F. Arbabifar, and F. I. Olness, Phys. Rev. D 83, 054017 (2011).

[45] S. A. Tehrani and A. N. Khorramian, J. High Energy Phys. 07 (2007) 048.

[46] A. N. Khorramian, A. Mirjalili, and S. A. Tehrani, J. High Energy Phys. 10 (2004) 062.

[47] S. A. Tehrani, F. Taghavi-Shahri, A. Mirjalili, and M. M. Yazdanpanah, Phys. Rev. D 87, 114012 (2013); 88, 039902(E) (2013).

[48] F. Taghavi-Shahri, H. Khanpour, S. A. Tehrani, and Z. Alizadeh Yazdi, Phys. Rev. D 93, 114024 (2016).

[49] H. Khanpour, S. T. Monfared, and S. A. Tehrani, Phys. Rev. D 96, 074037 (2017).

[50] H. Khanpour, S. T. Monfared, and S. A. Tehrani, Phys. Rev. D 95, 074006 (2017).

[51] A. Airapetian et al. (HERMES Collaboration), Phys. Rev. D 75, 012007 (2007).

[52] K. Ackerstaff et al. (HERMES Collaboration), Phys. Lett. B 404, 383 (1997).

[53] A. Airapetian et al. (HERMES Collaboration), Phys. Lett. B 442, 484 (1998).

[54] P. L. Anthony et al. (E142 Collaboration), Phys. Rev. D 54, 6620 (1996).

[55] K. Abe et al. (E154 Collaboration), Phys. Lett. B 405, 180 (1997).

[56] K. Abe et al. (E154 Collaboration), Phys. Rev. Lett. 79, 26 (1997).

[57] K. Abe et al. (E143 Collaboration), Phys. Rev. D 58, 112003 (1998).

[58] P. L. Anthony et al. (E155 Collaboration), Phys. Lett. B 463, 339 (1999).

[59] J. Ashman et al. (European Muon Collaboration), Nucl. Phys. B328, 1 (1989).

[60] P. L. Anthony et al. (E155 Collaboration), Phys. Lett. B 493, 19 (2000).

[61] J. Ashman et al. (European Muon Collaboration), Phys. Lett. B 206, 364 (1988).

[62] B. Adeva et al. (Spin Muon Collaboration), Phys. Rev. D 58, 112001 (1998).

[63] M. G. Alekseev et al. (COMPASS Collaboration), Phys. Lett. B 690, 466 (2010).

[64] E. S. Ageev et al. (COMPASS Collaboration), Phys. Lett. B 612, 154 (2005).

[65] V. Yu. Alexakhin et al. (COMPASS Collaboration), Phys. Lett. B 647, 8 (2007).
[66] K. V. Dharmawardane et al. (CLAS Collaboration), Phys. Lett. B 641, 11 (2006).

[67] X. Zheng et al. (Jefferson Lab Hall A Collaboration), Phys. Rev. C 70, 065207 (2004).

[68] C. Adolph et al. (COMPASS Collaboration), Phys. Lett. B 753, 18 (2016).

[69] C. Adolph et al. (COMPASS Collaboration), Phys. Lett. B 769, 34 (2017).

[70] W. Vogelsang, Phys. Rev. D 54, 2023 (1996).

[71] W. Furmanski and R. Petronzio, Z. Phys. C 11, 293 (1982).

[72] G. T. Bodwin and J.-W. Qiu, Phys. Rev. D 41, 2755 (1990).

[73] E. B. Zijlstra and W. L. van Neerven, Nucl. Phys. B417, 61 (1994); B501, 599(E) (1997).

[74] A. Vogt, Comput. Phys. Commun. 170, 65 (2005).

[75] M. Lacombe, B. Loiseau, R. Vinh Mau, J. Cote, P. Pires, and R. de Tourreil, Phys. Lett. 101B, 139 (1981).

[76] M. A. Ahmed and G. G. Ross, Nucl. Phys. B111, 441 (1976).

[77] R. Mertig and W. L. van Neerven, Z. Phys. C 70, 637 (1996).

[78] M. Gluck, E. Reya, and A. Vogt, Z. Phys. C 48, 471 (1990).

[79] J. Blumlein and A. Vogt, Phys. Rev. D 58, 014020 (1998).

[80] F. Arbabifar, A. N. Khorramian, and M. Soleymaninia, Phys. Rev. D 89, 034006 (2014).

[81] C. Amsler et al. (Particle Data Group), Phys. Lett. B 667, 1 (2008).

[82] K. A. Olive et al. (Particle Data Group), Chin. Phys. C 38, 090001 (2014).

[83] C. A. Aidala, S. D. Bass, D. Hasch, and G. K. Mallot, Rev. Mod. Phys. 85, 655 (2013).

[84] S. D. Bass and A. W. Thomas, Phys. Lett. B 684, 216 (2010).

[85] J. J. Ethier, N. Sato, and W. Melnitchouk, Phys. Rev. Lett. 119, 132001 (2017).

[86] F. James and M. Roos, Comput. Phys. Commun. 10, 343 (1975).

[87] C. Patrignani et al. (Particle Data Group), Chin. Phys. C 40, 100001 (2016).

[88] S. Taheri Monfared, Z. Haddadi, and A. N. Khorramian, Phys. Rev. D 89, 074052 (2014); 89, 119901(E) (2014).

[89] H. Khanpour, A. N. Khorramian, and S. A. Tehrani, J. Phys. G 40, 045002 (2013).

[90] B. Adeva et al. (Spin Muon Collaboration), Phys. Rev. D 58, 112002 (1998).

[91] A. Vafaee and A. N. Khorramian, Nucl. Phys. B921, 472 (2017).

[92] J. Blumlein, H. Bottcher, and A. Guffanti, Nucl. Phys. B774, 182 (2007).

[93] A. N. Khorramian and S. A. Tehrani, Phys. Rev. D 78, 074019 (2008).

[94] S. Alekhin, J. Blumlein, S. Klein, and S. Moch, Phys. Rev. D 81, 014032 (2010).

[95] M. Gluck, E. Reya, and C. Schuck, Nucl. Phys. B754, 178 (2006).

[96] A. D. Martin, W. J. Stirling, R. S. Thorne, and G. Watt, Eur. Phys. J. C 64, 653 (2009).

[97] F. D. Aaron et al. (ZEUS and H1 Collaborations), J. High Energy Phys. 01 (2010) 109.

[98] D. d'Enterria et al., Proceedings, High-Precision $\alpha_{s}$ Measurements from LHC to FCC-ee, (CERN, Geneva, 2015), arXiv:1512.05194. 
[99] A. N. Khorramian, H. Khanpour, and S. A. Tehrani, Phys. Rev. D 81, 014013 (2010).

[100] J. D. Bjorken, Phys. Rev. D 1, 1376 (1970).

[101] S. Wandzura and F. Wilczek, Phys. Lett. 72B, 195 (1977).

[102] A. Piccione and G. Ridolfi, Nucl. Phys. B513, 301 (1998).
[103] P. L. Anthony et al. (E155 Collaboration), Phys. Lett. B 553, 18 (2003).

[104] A. Airapetian et al. (HERMES Collaboration), Eur. Phys. J. C 72, 1921 (2012).

[105] D. Adams et al. (Spin Muon Collaboration), Phys. Rev. D 56, 5330 (1997).

[106] E. Leader, arXiv:1604.00305. 